\title{
Numerical solutions of compressible convection with an infinite Prandtl number: comparison of the anelastic and anelastic liquid models with the exact equations
}

\author{
Jezabel Curbelo ${ }^{1,2}$, Lucia Duarte ${ }^{3}$, Thierry Alboussière ${ }^{4}$, Fabien \\ Dubuffet $^{4}$, Stéphane Labrosse ${ }^{4}$, Yanick Ricard ${ }^{4}$ \\ ${ }^{1}$ Departamento de Matemáticas, Facultad de Ciencias, Universidad Autónoma de Madrid, \\ 28049, Madrid, Spain \\ ${ }^{2}$ Instituto de Ciencias Matemáticas, CSIC-UAM-UC3M-UCM, C/Nicolás Cabrera 15, Campus \\ Cantoblanco UAM, 28049, Madrid, Spain \\ ${ }^{3}$ Department of Physics and Astronomy, University of Exeter, UK \\ ${ }^{4}$ Université de Lyon, UCBL, ENSL, CNRS, LGL-TPE, 69622 Villeurbanne, France
}

(Received xx; revised $\mathrm{xx}$; accepted $\mathrm{xx}$ )

We developed a numerical method for the set of equations governing fully compressible convection in the limit of infinite Prandtl numbers. Reduced models have also been analyzed, such as the anelastic approximation and the anelastic liquid approximation. The tests of our numerical schemes against self-consistent criteria have shown that our numerical simulations are consistent from the point of view of energy dissipation, heat transfer and entropy budget. The equation of state of an ideal gas has been considered in this work. Specific effects arising because of the compressibility of the fluid are studied, like the scaling of viscous dissipation and the scaling of the heat flux contribution due to the mechanical power exerted by viscous forces. We analyzed the solutions obtained with each model (full compressible model, anelastic and anelastic liquid approximations) in a wide range of dimensionless parameters and determined the errors induced by each approximation with respect to the full compressible solutions. Based on a rationale on the development of the thermal boundary layers, we can explain reasonably well the differences between the full compressible and anelastic models, in terms of both the heat transfer and viscous dissipation dependence on compressibility. This could be mostly an effect of density variations on thermal diffusivity. Based on the different forms of entropy balance between exact and anelastic models, we find that a necessary condition for convergence of the anelastic results to the exact solutions is that the product $\epsilon q$ must be small compared to unity, where $\epsilon$ is the ratio of the superadiabatic temperature difference to the adiabatic difference and $q$ is the ratio of the superadiabatic heat flux to the heat flux conducted along the adiabat. The same condition seems to be also associated with a convergence of the computed heat fluxes. Concerning the anelastic liquid approximation, we confirm previous estimates by Anufriev et al. (2005) and find that its results become generally close to those of the full compressible model when $\alpha T \mathcal{D}$ is small compared to one, where $\alpha$ is the isobaric thermal expansion coefficient, $T$ the temperature (here $\alpha T=1$ for an ideal gas) and $\mathcal{D}$ the dissipation number.

Key words: compressible convection, thermal convection, anelastic approximation, anelastic liquid approximation. 


\section{Introduction}

Convection plays an important role for heat transfer in the deep interior of planets and stars, thus controlling their evolution. Many factors must be taken into account when modelling planetary and stellar convection: composition, rheology, heterogeneity of viscosity... Among them, in these large objects where the variation of density with pressure (or depth) is much larger than with temperature, compressibility lies at the heart of convection models and is strongly connected to the choice of a necessary convection approximation. It is sometimes not possible to use the original governing equations in a numerical model because they produce the whole class of acoustic waves, even though one is only interested in the much slower convection motion generated by buoyancy. The drastically simplified Oberbeck or Boussinesq model (Oberbeck 1879; Boussinesq 1903) suppresses the acoustic waves, which is favorable for numerical simulations, but fails to retrieve the so-called adiabatic temperature gradient in a nearly hydrostatic convecting fluid domain (except in Spiegel \& Veronis (1971) in the case of a small adiabatic gradient). Intermediate sound-proof models have been proposed, in particular the anelastic (Ogura \& Phillips 1961; Braginsky \& Roberts 1995; Lantz \& Fan 1999) and anelastic liquid (Anufriev et al. 2005) models. Our objective here is to better understand the exact governing equations, and describe and analyse the differences between the results obtained using these equations and those obtained using an anelastic or anelastic liquid model.

The question of the interplay between acoustic waves and convection is often taken as the key factor, although not the only one, for the applicability of the anelastic approximation (see for instance Verhoeven et al. 2015). This depends highly on the value of the Mach number, ratio of the typical convection speed to the acoustic wave celerity. However, the anelastic approximation differs from the full compressible model in different ways, and we concentrate in this paper on factors other than the question of the acoustic waves and the Mach number. This point of view is driven by geophysical applications: convection in the mantle, core and atmosphere of the Earth is characterized by a Mach number ranging from tiny to small. In the Earth's mantle, seismic P (acoustic) waves propagate at velocities of order $10 \mathrm{~km} \mathrm{~s}^{-1}$ while typical velocities are $0.1 \mathrm{~m} \mathrm{yr}^{-1}$, hence the Mach number is of order $10^{-12}$. In the liquid outer core of the Earth, the typical velocities inferred from geomagnetic variations are of order $10^{-4} \mathrm{~m} \mathrm{~s}^{-1}$ and seismic $\mathrm{P}$ waves have the same typical celerity of $10 \mathrm{~km} \mathrm{~s}^{-1}$ : the Mach number in the Earth's outer core is of order $10^{-8}$. In the atmosphere, sound velocities of a few $100 \mathrm{~m} \mathrm{~s}^{-1}$ compared to wind velocities of a few $10 \mathrm{~km} \mathrm{~s}^{-1}$ lead to Mach numbers of order $10^{-2}$. On the contrary, the situations that we consider do not apply to the upper atmosphere of Jupiter or Saturn, or to the surface of the Sun where convective velocities of several $\mathrm{km} \mathrm{s}^{-1}$ imply Mach number close to unity. To ensure a small Mach number, we have chosen to study the case of infinite Prandtl number convection: as shown in section 4, an infinite Prandtl number implies that the Mach number is zero.

In this paper, we make a further assumption that the equation of state is that of ideal gases. This equation of state introduces only one dimensionless parameter in the problem of convection, namely the ratio of heat capacities $\gamma$. We cannot argue that an ideal gas with infinite Prandtl number corresponds to a physical realization in geophysics or astrophysics. What we aim for is a coherent set of equations for convection, as simple as possible, so that approximate models of convection can be tested extensively and that some analytical predictions can be made. Although the ideal gas equation of state is a particular case, we believe that our results on the structure of compressible flows and on the validity of anelastic approximations remain useful for other equations of state. For 
instance, the relative influence of pressure and temperature on density depends simply on the dissipation parameter and on the ratio of heat capacities (Alboussière \& Ricard 2017), and those parameters can be specified arbitrarily using an ideal gas equation of state.

Within this simplified framework (no sound waves, ideal gas equation of state), compressible convection is governed by a few dimensionless numbers: a Rayleigh number, a dissipation parameter, a ratio of hot to cold imposed temperatures and a ratio of heat capacities. A first objective of this paper is to understand the specific role of compressibility (the dissipation parameter) in key outcomes, such as the heat flux transferred or the amount of energy dissipated by viscous stress. The second objective concerns the anelastic approximation models: when the temperature field is close to the adiabatic temperature profile, we would like to understand what is the parameter (or combination of the parameters listed above) indicating when an anelastic model is supposed to lead to results similar to those from the complete set of governing equations. Is that only related to the superadabatic parameter (ratio of superadiabatic temperature difference to the adiabatic temperature difference) or does it also involve the superadiabatic Rayleigh number?

The article is organized as follows. Section 2 describes the physical set-up and provides the governing equations as well as a detailed characterization of the adiabatic solution. Section 3 introduces the simplifications related to the choice of the perfect gas equation of state. Section 4 is devoted to a discussion of the Mach number at infinite Prandtl number and to the timescale of the viscous relaxation replacing sound waves. In section 5 , we briefly introduce the numerical methods used to obtain the solutions, we list the different forms of viscous energy dissipation, and obtain some estimates of the numerical errors. In section 6 , we show snapshots of the superadiabatic temperature field for various values of the governing parameters to give an idea of the different regimes explored in this study. The averaged temperature profiles are shown in section 7 , followed by a rationale in section 8 allowing us to make predictions on boundary layer thicknesses, heat transfer and entropy sources. The numerical results for different approximations are presented and compared in section 9 in terms of heat transfer and in section 10 in terms of viscous dissipation. Section 11 is devoted to entropy. In section 11.1, we show that the global entropy balance takes a different form in anelastic models, compared to the exact entropy balance. In section 11.4, we obtain upper and lower bounds for the "conduction" and "viscous dissipation" parts of the entropy sources in the anelastic approximation. Finally, we discuss the validity criteria for each approximation in section 12 .

\section{Physical set-up and governing equations}

The physical set-up consists in a two dimensional rectangular fluid layer $\Omega$ with uniform (dynamical) viscosity $\eta$, thermal conductivity $k$, and gravity $\mathrm{g}$ parallel to two sides (see Fig. 1) pointing towards the bottom of the page. The bottom and top sides, of length $L$, are maintained at constant temperatures, $T_{b o t}$ and $T_{t o p}$ respectively, and are subjected to free-slip, impermeable boundary conditions. The vertical sides, of length $d$, satisfy periodic boundary conditions. The geometry is defined through the aspect ratio $\Gamma=L / d$. The value $\Gamma=4$ will be most often used. Coordinates $(x, z)$ are defined such that $x$ is the horizontal coordinate $(0 \leqslant x \leqslant L)$ and $z$ the upward vertical coordinate $(0 \leqslant z \leqslant d)$, while $t$ is the variable of time. The average density in the rectangular domain is set to a value $\rho_{0}$ and an equation of state (see section 3 ) needs to be chosen for the fluid.

The exact governing equations in the framework of continuum mechanics are those of 
continuity, Stokes equation (infinite Prandtl number makes inertia negligible), entropy evolution and equation of state, for the density $\rho$, velocity field $\mathbf{v}$, specific entropy $s$, pressure $P$, temperature $T$ :

$$
\begin{aligned}
& \frac{\partial \rho}{\partial t}+\boldsymbol{\nabla} \cdot(\rho \mathbf{v})=0, \\
& \mathbf{0}=-\boldsymbol{\nabla} P+\rho \mathbf{g}+\boldsymbol{\nabla} \cdot \tau, \\
& \rho T\left[\frac{\partial s}{\partial t}+\mathbf{v} \cdot \boldsymbol{\nabla} s\right]=\dot{\varepsilon}: \tau+\nabla \cdot(k \boldsymbol{\nabla} T), \\
& T=T(\rho, s), \quad P=P(\rho, s),
\end{aligned}
$$

where the deformation rate tensor $\dot{\varepsilon}$ and stress tensor $\tau$ are defined as follows

$$
\dot{\varepsilon}_{i j}=\frac{1}{2}\left(\partial_{j} v_{i}+\partial_{i} v_{j}\right), \quad \tau_{i j}=\eta\left(\partial_{j} v_{i}+\partial_{i} v_{j}-\frac{2}{3} \delta_{i j} \boldsymbol{\nabla} \cdot \mathbf{v}\right),
$$

using Stokes hypothesis of zero bulk viscosity.

In stable cases (see Alboussière \& Ricard 2017), a motionless steady diffusive solution exists, characterized by a linear temperature profile $T_{b}(z)$, a hydrostatic pressure profile $P_{b}(z)$ and a density profile $\rho_{b}(z)$ such that

$$
T_{b}(z)=T_{b o t}-\frac{z}{d} \Delta T, \quad \frac{\mathrm{d} P_{b}}{\mathrm{~d} z}=-\rho_{b} g,
$$

where $\Delta T=T_{b o t}-T_{t o p}$ is the imposed temperature difference. For a given equation of state, a unique solution is obtained under the mass constraint:

$$
\rho_{0}=\frac{1}{d} \int_{0}^{d} \rho_{b}(z) \mathrm{d} z
$$

We also define an adiabatic (or isentropic) profile of temperature, pressure, density, coefficient of thermal expansion, heat capacity at constant pressure, $T_{a}(z), P_{a}(z), \rho_{a}(z)$, $\alpha_{a}(z)$ and $c_{p a}(z)$, characterized by a uniform entropy $s_{a}$ and hydrostatic conditions

$$
\frac{\mathrm{d} T_{a}}{\mathrm{~d} z}=-\frac{\alpha_{a} g T_{a}}{c_{p a}}, \quad \frac{\mathrm{d} P_{a}}{\mathrm{~d} z}=-\rho_{a} g
$$

The adiabatic profile must also satisfy the equation of state. We prescribe the adiabatic profile to satisfy the mass constraint $(2.7)$, with $\rho_{a}$ instead of $\rho_{b}$. This is however not sufficient to define a unique profile, so we make the additional choice of equal superadiabatic departures from the thermal boundary conditions: $T_{\text {bot }}-T_{a}(0)=T_{a}(d)-T_{\text {top }}$. In highly convective cases, it is expected that the solutions should converge toward the adiabatic profile, although we will see that the actual temperature may be shifted away from a profile with symmetrical superadiabatic temperature differences. Independently of these expectations, this adiabatic profile will be used as a reference to expand thermodynamic quantities in the anelastic models: $T=T_{a}+T^{\prime}$ and similarly for the other variables of state. The adiabatic temperature difference across the layer is denoted $\Delta T_{a}=T_{a}(0)-T_{a}(d)$, while the superadiabatic temperature difference is denoted $\Delta T_{s a}=\Delta T-\Delta T_{a}=T_{b o t}-T_{a}(0)+T_{a}(d)-T_{\text {top }}$.

Let us now express a dimensionless version of the problem. We choose the arithmetic average of top and bottom temperatures, $T_{0}=\left(T_{t o p}+T_{b o t}\right) / 2$ as a reference temperature. We have already defined $\rho_{0}$ as the average density of the fluid layer. Through the equation of state, we can then obtain a reference value, denoted with a subscript 0 , 
for any thermodynamic quantity: pressure $P_{0}$, heat capacity at constant pressure $c_{p 0}$, coefficient of thermal expansion $\alpha_{0} \ldots$ The variables of time $t$, spatial coordinates $(x, z)$, velocity $\mathbf{v}$, temperature $T$, pressure $P$, entropy $s$, deformation tensor $\dot{\varepsilon}$, stress tensor $\tau$ are made dimensionless using the following scales respectively, $\rho_{0} c_{p 0} d^{2} / k, d, k /\left(\rho_{0} c_{p 0} d\right)$, $T_{0}, \rho_{0} c_{p 0} T_{0}, c_{p 0}, k /\left(\rho_{0} c_{p 0} d^{2}\right)$ and $k \eta /\left(\rho_{0} c_{p 0} d^{2}\right)$. Basically, the timescale is that of thermal diffusion across the height $d$ of the cavity (the lengthscale), velocity and tensor of deformation rate are scaled using that timescale and lengthscale. The scale for the stress tensor is obtained by multiplying the scale of deformation tensor rate by viscosity. It could also have been the pressure scale, however the scale for pressure is built from that of temperature $T_{0}$ (mean value of top and bottom temperatures) as a thermal energy per unit volume $\rho_{0} c_{p 0} T_{0}$. Entropy is simply scaled by the specific heat $c_{p 0}$. The governing equations $(2.1),(2.2),(2.3),(2.4)$ and $(2.5)$ are now written in a dimensionless form:

$$
\begin{aligned}
\frac{\partial \rho}{\partial t}+\nabla \cdot(\rho \mathbf{v}) & =0 \\
\mathbf{0} & =-\frac{R \hat{\alpha}}{\mathcal{D}} \boldsymbol{\nabla} P-R \rho \mathbf{e}_{z}+\nabla \cdot \tau, \\
\rho T\left[\frac{\partial s}{\partial t}+\mathbf{v} \cdot \nabla s\right] & =\frac{\mathcal{D}}{R \hat{\alpha}} \dot{\varepsilon}: \tau+\nabla^{2} T, \\
T=T(\rho, s), \quad P=P(\rho, s), & \quad \tau_{i j}=\partial_{j} v_{i}+\partial_{i} v_{j}-\frac{2}{3} \delta_{i j} \boldsymbol{\nabla} \cdot \mathbf{v},
\end{aligned}
$$

where the dimensionless numbers $R, \hat{\alpha}$ and dissipation number $\mathcal{D}$ are defined as follows:

$$
\begin{aligned}
& R=\frac{\rho_{0}^{2} c_{p 0} g d^{3}}{k \eta}, \\
& \hat{\alpha}=\alpha_{0} T_{0}, \\
& \mathcal{D}=\frac{\alpha_{0} g d}{c_{p 0}} .
\end{aligned}
$$

We also need to define an extra dimensionless number to set the temperature boundary condition. We choose the temperature ratio $r$ between the bottom and top boundaries:

$$
r=\frac{T_{b o t}}{T_{t o p}} .
$$

The dimensionless thermal boundary conditions can then be expressed as:

$$
T_{b o t}=\frac{2 r}{1+r} \quad T_{\text {top }}=\frac{2}{1+r} .
$$

The dimensionless parameters introduced so far, $R, \hat{\alpha}, \mathcal{D}$ and $r$ are enough to describe the physical problem, although other parameters may appear when an equation of state is specified, like for instance $\gamma=c_{p 0} / c_{v 0}$ the ratio of specific heat capacities at the reference conditions.

The dimensionless number $R$ can be related to the proper superadiabatic Rayleigh number $R a_{s a}$ based on the superadiabatic temperature difference $\Delta T_{s a}$. It can also be related to the adiabatic temperature difference $\Delta T_{a}$ or to $\Delta T$, using another dimensionless parameter $\epsilon$ measuring the relative amplitude of the superadiabatic to the adiabatic 
temperature difference:

$$
\begin{aligned}
\epsilon & =\frac{\Delta T_{s a}}{\Delta T_{a}}=\frac{\delta T_{s a}}{\delta T_{a}} \\
R a_{s a} & =R \hat{\alpha} \delta T_{s a}=R \hat{\alpha} \epsilon \delta T_{a}=R \hat{\alpha} \frac{\epsilon}{1+\epsilon} \delta T,
\end{aligned}
$$

where $\delta T_{a}=\Delta T_{a} / T_{0}, \delta T_{s a}=\Delta T_{s a} / T_{0}, \delta T=\Delta T / T_{0}$ are the dimensionless adiabatic, superadiabatic and total temperature differences. The dimensionless temperature difference is easily related to the temperature ratio: $\delta T=2(r-1) /(r+1)$. It is also equal to the dimensionless temperature gradient of the conduction profile (with constant thermal conductivity), which was called $a$ in a previous paper (Alboussière \& Ricard 2017), so that the dimensionless counterpart of the temperature profile in (2.6) is

$$
T_{b}(z)=1-\delta T\left(z-\frac{1}{2}\right)=1-a\left(z-\frac{1}{2}\right) .
$$

For any general equation of state, the dimensionless adiabatic temperature difference is of the order of the dissipation number $\mathcal{D}$ and for ideal gases one has exactly $\delta T_{a}=\mathcal{D}$. In physical terms, the dissipation parameter $\mathcal{D}$ is related to adiabatic heating (resp. cooling) on compression (resp. decompression). It is a measure of the temperature difference due to the adiabatic gradient over the height of the system, divided by the mean temperature. With the definitions above, it is hence possible to specify $R a_{s a}$ instead of $R$, or $\epsilon$ instead of $r$ in the list of dimensionless parameters as $R=R a_{s a} /(\hat{\alpha} \in \mathcal{D})$ and $r=[2+\mathcal{D}(1+$ $\epsilon)] /[2-\mathcal{D}(1+\epsilon)]$. As a final point on dimensionless parameters, it must be noted that, for a fixed temperature ratio $r$, there exists a maximal value of the dissipation number $\mathcal{D}_{\max }$ above which the fluid is stably stratified, and $\epsilon$ can be expressed as a function of $\mathcal{D}$ (and conversely)

$$
\begin{aligned}
\mathcal{D}_{\text {max }} & =2 \frac{r-1}{r+1}, \\
\epsilon & =\frac{\mathcal{D}_{\text {max }}-\mathcal{D}}{\mathcal{D}}, \\
\mathcal{D} & =\frac{\mathcal{D}_{\text {max }}}{1+\epsilon} .
\end{aligned}
$$

For instance, when $r=3$, we have $\mathcal{D}_{\max }=1$, and when $\mathcal{D}$ approaches this maximum value, $\epsilon$ approaches zero.

The governing equations presented above (2.9), (2.10), (2.11), (2.12) and (2.13) are those derived from the general dynamical and thermodynamical principles applied to a fluid, which we call here the full compressible model FC. We are also writing, on table 1, the governing equations associated with approximate models of those general equations: the anelastic approximation AA (Ogura \& Phillips 1961; Braginsky \& Roberts 1995; Lantz \& Fan 1999), anelastic liquid approximation ALA (Anufriev et al. 2005) and Boussinesq model B (Oberbeck 1879; Boussinesq 1903). In this work, we shall compare the results of the full compressible FC, anelastic approximation AA and anelastic liquid approximation ALA. The anelastic equations AA and ALA have been obtained using the same scales as those used to obtain the dimensionless equations of the full compressible model FC. The Boussinesq model B is shown here for comparison, although we shall not run Boussinesq numerical calculations: temperature is scaled using the total imposed temperature difference $T_{b o t}-T_{t o p}$ for this model only. Notice that the limit of the FC, AA or ALA approximations when $\mathcal{D}$ tends to zero is not the Boussinesq approximation. The latter implies in addition that the temperature difference across the convecting domain 


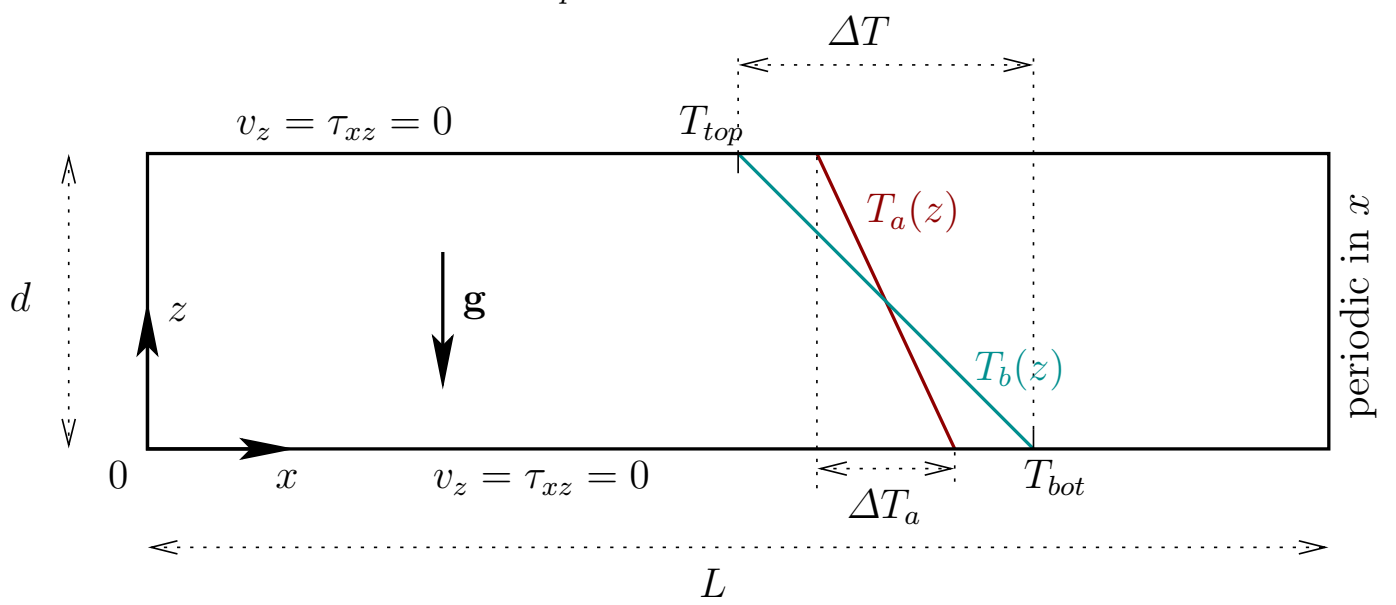

Figure 1. Rectangular, 2D, physical setup $\Omega$. The boundary conditions are periodic in the horizontal $x$ direction. Stress-free, impermeable conditions apply on bottom and top boundaries, held at imposed hot $T_{b o t}$ and cold $T_{t o p}$ temperatures.

is negligible compared to the average thermodynamic temperature which is not the case in our simulations. The order of these limits matter: the Boussinesq approximation corresponds to $\lim _{r \rightarrow 1} \lim _{\mathcal{D} \rightarrow 0}$ (see also Alboussière \& Ricard (2017)).

It is worth noting that the ratio $r$ is irrelevant in anelastic models (AA and ALA). This can be proven by changing the dimensional scales for temperature, pressure and entropy fluctuations $T^{\prime}, P^{\prime}$ and $s^{\prime}$ for $\Delta T_{s a}, \rho_{0} c_{p 0} \Delta T_{s a}$ and $c_{p 0} \Delta T_{s a} / T_{0}$ (instead of $T_{0}, \rho_{0} c_{p 0} T_{0}$ and $\left.c_{p 0}\right)$. Then, $R a_{s a}$ and $\mathcal{D}$ are the only dimensionless parameters in the Stokes and entropy equations. Moreover, the dimensionless thermal boundary conditions become simply $T^{\prime}= \pm 1 / 2$ at $z=0$ and $z=1$. Hence the anelastic solutions are independent of $r$. This result can also be inferred from the original derivation of the anelastic equations Ogura \& Phillips (1961), since they are obtained by linearization of the thermodynamic functions about the hydrostatic adiabatic state: the role of the superadiabatic temperature difference $\Delta T_{s a}$ is only to contribute to the superadiabatic Rayleigh number.

\section{Equation of State}

The equation of state of ideal gases, $P / \rho=\mathcal{R} T / M$, where $\mathcal{R}$ is the universal gas constant and $M$ the molar mass of the gas, with $c_{p}=c_{p_{0}}, c_{v}=c_{v 0}$ and $\gamma=c_{p_{0}} / c_{v 0}$ constants, is written using our dimensionless variables

$$
\rho=\frac{P}{\left(1-\gamma^{-1}\right) T} .
$$

From this equation of state, $P$ and $T$ in terms of $\rho$ and $s$ take the following dimensionless forms

$$
\begin{aligned}
& T=\exp (\gamma s) \rho^{\gamma-1}, \\
& P=\left(1-\gamma^{-1}\right) \exp (\gamma s) \rho^{\gamma} .
\end{aligned}
$$




\begin{tabular}{|c|c|c|c|}
\hline & continuity & entropy & momentum \\
\hline $\mathrm{FC}$ & $\frac{\partial \rho}{\partial t}+\boldsymbol{\nabla} \cdot(\rho \mathbf{v})=0$ & $\rho T \frac{\mathrm{D} s}{\mathrm{D} t}=\frac{\mathcal{D}}{R \hat{\alpha}} \dot{\varepsilon}: \tau+\nabla^{2} T$ & $-\frac{R \hat{\alpha}}{\mathcal{D}} \nabla P-R \rho \mathbf{e}_{z}+\nabla \cdot \tau=0$ \\
\hline $\mathrm{AA}$ & \multirow[t]{2}{*}{$\boldsymbol{\nabla} \cdot\left(\rho_{a} \mathbf{v}\right)=0$} & $\rho_{a} T_{a} \frac{\mathrm{D} s^{\prime}}{\mathrm{D} t}=\frac{\mathcal{D}}{R \hat{\alpha}} \dot{\varepsilon}: \tau+\nabla^{2} T$ & $-\frac{R \hat{\alpha}}{\mathcal{D}} \rho_{a} \boldsymbol{\nabla}\left(\frac{P^{\prime}}{\rho_{a}}\right)+R \hat{\alpha} \frac{\alpha_{a} \rho_{a} T_{a}}{{ }^{c_{p_{a}}}} s^{\prime} \mathbf{e}_{z}+\boldsymbol{\nabla} \cdot \tau=0$ \\
\hline ALA & & $\rho_{a} \frac{\mathrm{D}\left(c_{p_{a}} T^{\prime}\right)}{\mathrm{D} t}=-\mathcal{D} \alpha_{a} \rho_{a} v_{z} T^{\prime}+\frac{\mathcal{D}}{R \hat{\alpha}} \dot{\varepsilon}: \tau+\nabla^{2} T$ & $-\frac{R \hat{\alpha}}{\mathcal{D}} \rho_{a} \boldsymbol{\nabla}\left(\frac{P^{\prime}}{\rho_{a}}\right)+R \hat{\alpha} \alpha_{a} \rho_{a} T^{\prime} \mathbf{e}_{z}+\nabla \cdot \tau=0$ \\
\hline B & $\boldsymbol{\nabla} \cdot \mathbf{v}=0$ & $\frac{\mathrm{D} T}{\mathrm{D} t}=\nabla^{2} T$ & $-\boldsymbol{\nabla} P+R a_{s a} T \mathbf{e}_{z}+\nabla^{2} \mathbf{v}=0$ \\
\hline
\end{tabular}

TABle 1. Dimensionless equations. FC Full compressible model; AA Anelastic approximation model; ALA Anelastic Liquid approximation model; B Boussinesq approximation model. D/D $t$ stands for $\partial_{t}+\mathbf{v} \cdot \boldsymbol{\nabla}$.

We can also express entropy from temperature and pressure

$$
s=\ln T-\left(1-\gamma^{-1}\right) \ln \left(\frac{P}{1-\gamma^{-1}}\right),
$$

where the dimensionless entropy has been set arbitrarily to zero for the reference conditions $\rho=1$ and $T=1$. This equation of state implies also that the product $\alpha T$ is unity: $\hat{\alpha}=\alpha_{0} T_{0}=1$.

In the anelastic approximations, we need to derive the expression for the adiabatic reference profile and to express the perturbations of thermodynamical variables in terms of the variables chosen to describe the state of the fluid, namely $s$ and $\rho$ for the general anelastic approximation and $T$ for the anelastic liquid approximation. The adiabatic (or isentropic) profile, as defined by the equations of the adiabatic gradient and hydrostatic equilibrium (2.8) takes the following dimensionless expression for ideal gases:

$$
\begin{aligned}
& T_{a}(z)=1-\mathcal{D}\left(z-\frac{1}{2}\right) \\
& \rho_{a}(z)=\frac{\mathcal{D} /\left(1-\gamma^{-1}\right)}{(1+\mathcal{D} / 2)^{\frac{\gamma}{\gamma-1}}-(1-\mathcal{D} / 2)^{\frac{\gamma}{\gamma-1}}} T_{a}(z)^{\frac{1}{\gamma-1}}, \\
& P_{a}(z)=\frac{\mathcal{D}}{(1+\mathcal{D} / 2)^{\frac{\gamma}{\gamma-1}}-(1-\mathcal{D} / 2)^{\frac{\gamma}{\gamma-1}}} T_{a}(z)^{\frac{\gamma}{\gamma-1}} .
\end{aligned}
$$

As stated in section 1 this profile satisfies not only the hydrostatic adiabatic conditions, but also the mass constraint $\int_{0}^{1} \rho_{a}(z) d z=1$ and the condition of equal superadiabatic temperature difference at the top and bottom of the cavity. When Gibbs equation $T \mathrm{~d} s=$ $c_{p} \mathrm{~d} T-\rho^{-1} \mathrm{~d} P$ is made dimensionless and linearized around the adiabatic profile, linear 
relationships can be derived between the perturbations of thermodynamical variables

$$
\begin{aligned}
s^{\prime} & =\gamma^{-1} \frac{T^{\prime}}{T_{a}}-\left(1-\gamma^{-1}\right) \frac{\rho^{\prime}}{\rho_{a}}, \\
s^{\prime} & =\frac{T^{\prime}}{T_{a}}-\left(1-\gamma^{-1}\right) \frac{P^{\prime}}{P_{a}}, \\
\rho^{\prime} & =-\rho_{a} s^{\prime}+\gamma^{-1} \frac{\rho_{a}}{P_{a}} P^{\prime}, \\
T^{\prime} & =T_{a} s^{\prime}+\left(1-\gamma^{-1}\right) \frac{T_{a}}{P_{a}} P^{\prime} .
\end{aligned}
$$

\section{The Mach number at infinite $\operatorname{Pr}$ number}

The Mach number is the ratio between the fluid velocity and the celerity of sound waves. There may be some arbitrariness in the particular choice of velocity or celerity of sound but we shall see here that it will always lead to a Mach number equal to zero for an infinite Prandtl number. Let us first make clear that we are not just considering large values of the Prandtl number, but its infinite limit. In terms of dimensional parameters, the governing parameters $R, \hat{\alpha}$ and $\mathcal{D}$ can be determined using finite values of the dimensional parameters except for the thermal conductivity $k$ and viscosity $\eta$. For these last two parameters, only their product is assumed to be finite. Then their ratio $\eta / k$ (or rather $\operatorname{Pr}=\eta c_{p} / k$ ) is made larger and larger, i.e. its limit towards infinity is taken. The consequence of this choice is to eliminate inertia in the momentum equation. The infinite Prandtl number is not in the list of parameters and a numerical calculation of the velocity field will result in a finite factor times the velocity scale. The celerity of sound waves is $\sqrt{(\gamma-1) c_{p} T_{0}}$ for a perfect gas. Then, irrespectively of its exact definition, the Mach number will be a finite factor times the following scale $M$ :

$$
M=\frac{k /\left(\rho_{0} c_{p} d\right)}{\sqrt{(\gamma-1) c_{p} T_{0}}}=\operatorname{Pr}^{-1 / 2}\left(\frac{\mathcal{D}}{(\gamma-1) R}\right)^{1 / 2} .
$$

As $R, \gamma-1$ and $\mathcal{D}$ are finite and $\operatorname{Pr}$ infinite, the Mach number $M$ is zero.

Sound waves are thus absent from numerical solutions at infinite Prandtl number. However, it does not mean that the only relevant timescale is imposed by advection. Let us consider a fluid with uniform properties $T_{0}, P_{0}, \rho_{0}$ in a medium without gravity, and a localized perturbation $T^{\prime}, P^{\prime}, \rho^{\prime}$. This perturbation that we assume for simplicity to be only a function of $x$, relaxes and its evolution is controlled by the mass conservation $(2.9)$

and the momentum equilibrium

$$
\frac{\partial \rho^{\prime}}{\partial t}+\rho_{0} \frac{\partial v_{x}}{\partial x}=0
$$

$$
-P^{\prime}+\frac{4}{3} \eta \frac{\partial v_{x}}{\partial x}=0
$$

Let us further assume that the relaxation is fast enough to be adiabatic so that

$$
0=\frac{P^{\prime}}{P_{0}}-\gamma \frac{\rho^{\prime}}{\rho_{0}}
$$

Using (4.2), (4.3) and (4.4), we obtain that the perturbation decreases as

$$
\frac{\partial \rho^{\prime}}{\partial t}=-\frac{\rho^{\prime}}{t_{r}} \quad \text { with } \quad t_{r}=\frac{4}{3} \frac{\eta}{\gamma P_{0}} .
$$


The viscous isentropic relaxation timescale $t_{r}$ is the time necessary to expand (resp. contract) regions of excess (resp. deficit) pressure against viscous constraints. The timestep used in explicit numerical schemes must be shorter than this relaxation timescale to avoid the appearance and amplification of spurious fluctuations. The assumption of an adiabatic relaxation can now be checked by comparing $t_{r}$ to a thermal diffusion timescale $t_{d}=l^{2} \rho_{0} c_{p} / k$ for a lengthscale $l$. The ratio of the diffusion timescale to the relaxation time $t_{r}$ can be expressed using our dimensionless parameters

$$
\frac{t_{d}}{t_{r}}=\frac{3}{4}(\gamma-1) \frac{R}{\mathcal{D}}\left(\frac{l}{d}\right)^{2} .
$$

When $R$ is large, or $\mathcal{D}$ small, there exists a range of lengthscales $l$ (from the large scale $d$ down to a minimum scale $l_{\text {min }}$ corresponding to $t_{d} / t_{r} \sim 1$ ) for which the diffusion time is larger than the relaxation time, hence justifying the adiabatic assumption. For a given Rayleigh number, full compressible computations at small $\mathcal{D}$ take much more time than at large $\mathcal{D}$ as they must be performed with a time step small enough to resolve this viscous isentropic relaxation.

\section{Numerical methods}

To test the differences between different approximations, the equations are solved using a finite volume discretization on a staggered grid. The numerical methods used for the different approximations that appear at Table 1 have some differences, so we will explain the scheme for the fully compressible case and then give some pointers to solve the others.

For the full compressible case, at each time-step, the density $\rho$ and entropy $s$ are updated according to the continuity (2.9) and entropy (2.11) equations, using the velocity and temperature fields $\left(v_{x}, v_{z}\right)$ and $T$ from the previous time-step. The time-stepping method is the ADI method (Alternating Direction Implicit). The equations are discretized in two steps, where each step solves for one direction alone, first in the $x$ direction, then in the $z$ direction. The advantage of this method is that the equations in each step have a simpler structure and can be solved efficiently with a tridiagonal matrix algorithm. The boundary conditions for entropy are set from the physical boundary conditions on temperature $T$ and from the value of pressure $P$ at the previous time-step. Then, pressure $P$ and temperature $T$ are computed from the new density $\rho$ and entropy $s$ using the equation of state for ideal gases (3.3) and (3.2). Finally, the velocity field $\left(v_{x}, v_{z}\right)$ is updated knowing $P$ and $\rho$ by solving Stokes' equation (2.10). This is achieved using UMFPACK (Unsymmetric MultiFrontal method, Davis 2004, 2006). This modern inversion package allows this inversion to be extremely fast without the requirement to save all the pre-inversion coefficients and then all the elements of the resulting sparse matrix. Moreover, on a fixed grid with a constant dynamic viscosity, the LU factorisation has to be performed only once at the beginning, the solve step remaining at each time step being performed very efficiently.

The numerical scheme for the anelastic approximation model is subtly different because the general continuity equation is replaced by its zeroth-order expansion which does not depend on time. Therefore the scheme in this case is as follows at each time-step: first, we obtain the entropy perturbation $s^{\prime}$ by solving the entropy equation from the previous time-step using, as before, the ADI method for the time discretization. Temperature boundary conditions are imposed on $s^{\prime}$ (expressed from (3.9)), using the pressure field $P^{\prime}$ from the previous timestep. Secondly, we solve the linear system composed of Stokes and continuity equations in order to compute simultaneously the velocity vector field $\left(v_{x}, v_{z}\right)$ and pressure perturbations $P^{\prime}$. The source term is the entropy perturbation $s^{\prime}$ 
obtained just before. However, the solution to the pressure perturbation is not unique because only the gradient of $P^{\prime} / \rho_{a}$ is relevant. There is a single free parameter that we have to specify and we use this opportunity to enforce mass conservation: the pressure perturbations $P^{\prime}$ and the previously computed entropy perturbations $s^{\prime}$ must be such that

$$
\int_{\Omega} \rho^{\prime}(x, z, t) d x d z=0,
$$

using the linearized equation of state, whereby $\rho^{\prime}$ is a function of $s^{\prime}$ and $P^{\prime}$. Stokes, continuity and (5.1) are solved simultaneously as a single linear system. Finally, temperature $T^{\prime}$ and density $\rho^{\prime}$ perturbations are updated knowing $s^{\prime}$ and $P^{\prime}$ using equations (3.10) and (3.11).

The numerical scheme for the Anelastic Liquid approximation is simpler because we only need to compute $P^{\prime}, T^{\prime}$ and the velocity vector field, i.e. we only need the equation of state to impose that the mass remains constant according to equation (5.1). Here, $T^{\prime}$ is calculated from the entropy equation using the velocity field $\left(v_{x}, v_{z}\right)$ at the previous time-step. Then, to compute the velocity vector field $\left(v_{x}, v_{z}\right)$ and $P^{\prime}$, we solve the linear system composed of the Stokes and continuity equations using the new $T^{\prime}$. Again, the field $P^{\prime} / \rho_{a}$ is defined up to an additive constant from Stokes equation. This is precisely where the mass constraint (5.1) is used: that additive constant is adjusted such that the integral of $\rho^{\prime}$ (computed using the linearized equation of state from $P^{\prime}$ and $T^{\prime}$ ) vanishes. This determination of $P^{\prime}$ is only useful if we need that field, as it does not affect the determination of the other fields (contrary to the case of the AA approximation).

Our simulations can only reach moderate superadiabatic Rayleigh numbers from critical to 1000 times critical where we use a maximum number of 256 vertical nodes and 1024 horizontal nodes.

In order to assess the good performance and consistency of this numerical method with theoretical results (Hewitt et al. 1975a; McKenzie \& Jarvis 1980; Verhoogen 1980) the code is tested against self-consistent criteria of energy dissipation (Alboussière \& Ricard 2013). In the full compressible model FC, the following expressions of the viscous dissipation can be found to be equal, from the Stokes (2.10) and entropy (2.11) equations:

$$
\begin{aligned}
\frac{\mathcal{D}}{R}\langle\dot{\varepsilon}: \tau\rangle & =\langle\mathbf{v} \cdot \nabla P\rangle=\langle P \nabla \cdot \mathbf{v}\rangle=-\left\langle\frac{D P}{D t}\right\rangle \\
& =\left\langle\rho T \frac{D s}{D t}\right\rangle=-\left\langle\rho s \frac{D T}{D t}\right\rangle
\end{aligned}
$$

where $\langle\cdot\rangle$ denotes time-averaged volume integral. These equalities hold for any statistically steady solution of the equations, when the time-average is taken over a sufficiently long duration. Figure 2 shows that although these equalities are not imposed in the numerical simulations the relative differences between them turn out to be small in all cases, of order $10^{-6}$.

In the anelastic approximation AA, remembering $\hat{\alpha}=1$ for an ideal gas, the integrated viscous dissipation can be expressed as

$$
\langle\dot{\varepsilon}: \tau\rangle=R\left\langle\rho_{a} v_{z} s^{\prime}\right\rangle=R\left\langle\alpha_{a} \rho_{a} v_{z} T^{\prime}\right\rangle-R\left\langle\alpha_{a} v_{z} P^{\prime}\right\rangle,
$$

while in the anelastic liquid approximation ALA, this becomes simply

$$
\langle\dot{\varepsilon}: \tau\rangle=R\left\langle\alpha_{a} \rho_{a} v_{z} T^{\prime}\right\rangle .
$$

The expressions are shown to be numerically equivalent (see Fig. 2) within a relative error always smaller than $10^{-5}$. 


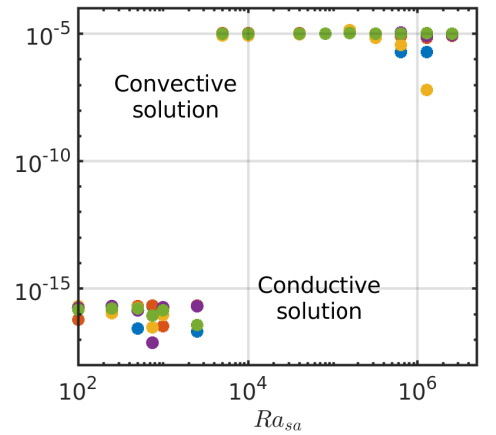

(a) Full compressible model

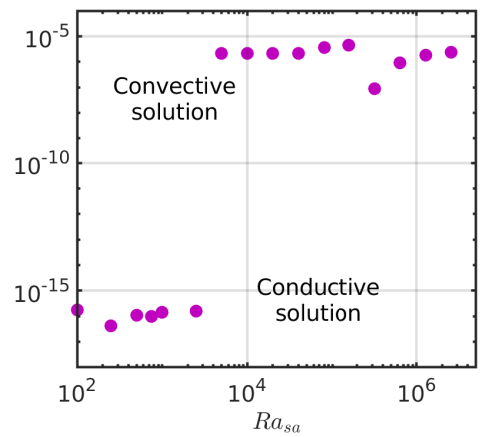

(c) Anelastic Liquid Approximation

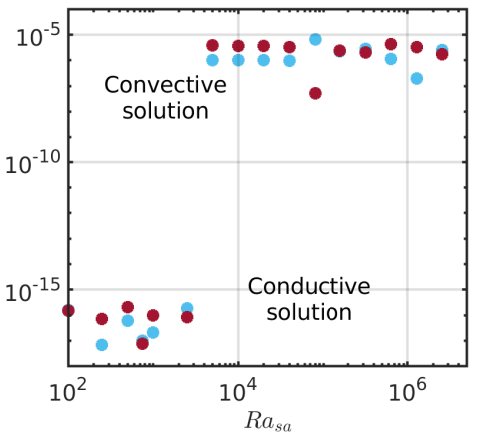

(b) Anelastic Approximation

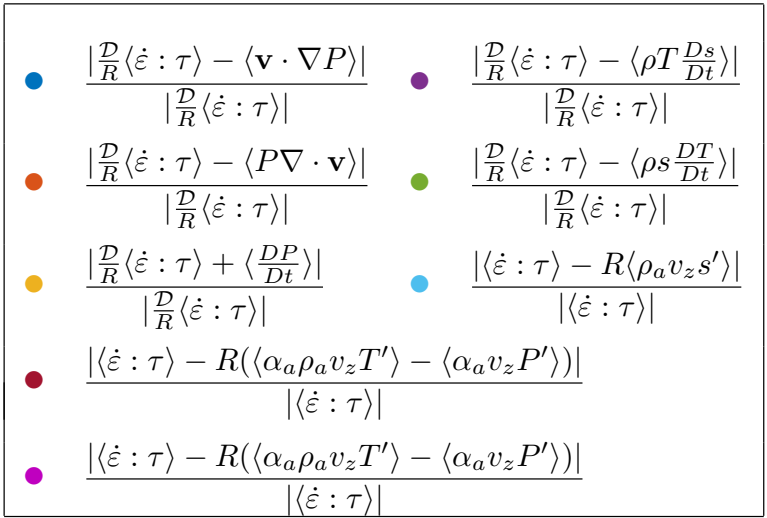

FiguRE 2. Computed differences between equivalent expressions for the viscous dissipation given by equation (5.2) for full-compressible solutions and by equations (5.3) and (5.4) for anelastic solutions, for $r=3, \gamma=1.4$ and $D=0.8(\epsilon=0.25)$.

We also test our numerical methods against self-consistent criteria of heat transfer. Integrating horizontally and time-averaging (2.11), using (2.9), (2.10) and Gibbs equation $\mathrm{d} h=T \mathrm{~d} s+\mathrm{d} P / \rho$, it can be shown that the dimensionless heat flux,

$$
Q(z)=\overline{\rho h v_{z}}-\frac{\mathcal{D}}{R} \overline{v_{j} \tau_{z j}}-\overline{\partial_{z} T},
$$

is conserved along the vertical direction, where - denotes time-averaged horizontal integral and $h$ is the specific enthalpy or Gibbs free energy (simply $c_{p 0} T$ for an ideal gas), made dimensionless using $c_{p 0} T_{0}$. The heat flux is the sum of three components: transport of enthalpy $\overline{\rho h v_{z}}$, shear-stress power $-\mathcal{D} / R \overline{v_{j} \tau_{z j}}$ and conduction $-\overline{\partial_{z} T}$. The first two components together form the 'convective flux'. In the anelastic and anelastic liquid approximations the heat flux takes the form

$$
Q(z)=\overline{\rho_{a} h^{\prime} v_{z}}-\frac{\mathcal{D}}{R} \overline{v_{j} \tau_{z j}}-\overline{\partial_{z} T}
$$

which only differs from $(5.5)$ because $\rho_{a} h^{\prime}$ replaces $\rho h$ in the first term (the perturbation of enthalpy $h^{\prime}$ is simply $T^{\prime}$ for an ideal gas). The computed heat flux is indeed constant in $z$, and its value simply denoted $Q$, is shown, for the full compressible case, when the dissipation number $\mathcal{D}$ is varied; see Figure 3 . However this value is slightly different depending on the model that has been used to calculate the solution as can be seen in 


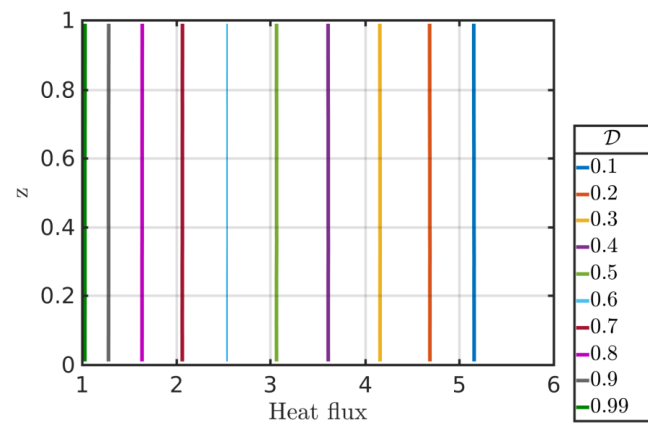

(a) Several $\mathcal{D}$ (Full compressible model)

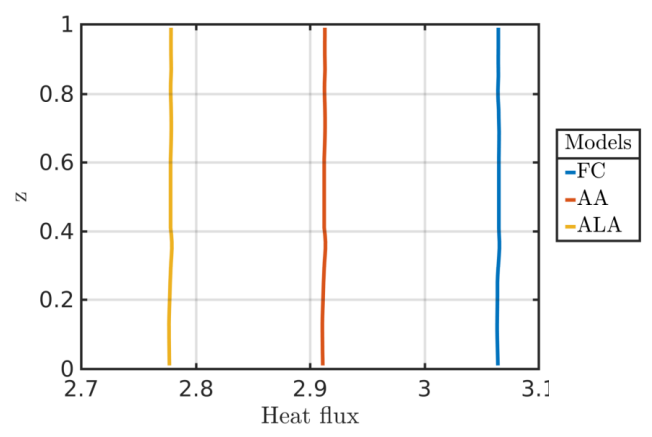

(b) Several models $(\mathcal{D}=0.5)$

Figure 3. Heat flux profile along the vertical direction, averaged over the horizontal direction $x$ and time, for $r=3$ and $R a_{s a}=10000$.

Figure 3 ( $\approx$ between $5 \%$ and $10 \%$ lower when approximate equations are used). This fact will be used to compare the solutions obtained with different methods in section 9 .

\section{Visualization of convection numerical models}

Before we analyze quantitatively the outcomes of our numerical models in the following sections, let us first look at temperature and velocity fields obtained using different models and approximations, and for different values of the governing parameters: small and large superadiabatic Rayleigh numbers, small and large values of the dissipation parameter.

Some snapshots of the convection are shown in Fig. 4 for two values of the superadiabatic Rayleigh number, $\mathcal{D}=0.6, r=3, \gamma=1.4$ and full compressible model FC, anelastic approximation AA and anelastic liquid approximation ALA. Small arrows indicate the velocity field while the background color corresponds to temperature minus the adiabatic profile (3.5). At $R a_{s a}=2 \times 10^{4}$, all flows are steady with two convective cells of the same size in the $2 \mathrm{D}$ cavity of aspect ratio 4 . This pattern is denoted by a full black dot. Full symbols denote steady convection. These symbols, full or empty, black or colored, will be used later to refer to the various convection patterns in several phase diagrams. However, there are subtle differences between FC, AA and ALA even at $R a_{s a}=2 \times 10^{4}$ : the background temperature is hotter for AA (and ALA to a lesser extent). This is most probably due to the asymmetry of the thermal boundary layers, as discussed in section 8. At $R a_{s a}=4 \times 10^{4}$, the pattern is unchanged for the FC flow but it has changed for AA (two convective cells of different size, full blue dot) and ALA (one big cell, full green dot). That change in pattern implies also a change in heat transfer. At slightly larger $R a_{s a}$ the transition to unsteady convection will also occur for different values of $R a_{s a}$ depending on the model of convection.

Full compressible calculations at larger values of the Rayleigh number, $R a_{s a}=3.2 \times$ $10^{5}$ and $R a_{s a}=1.28 \times 10^{6}$, and for two values of the dissipation number, $\mathcal{D}=0.2$ and $\mathcal{D}=0.9$, are shown in Fig. 5 , for a temperature ratio $r=3$ and $\gamma=1.4$. The corresponding solutions are all unsteady. The structures of temperature become thinner and more chaotic at large $R a_{s a}$. It can be seen also that the bulk of the fluid is shifted toward hot values of superadiabatic temperature for $\mathcal{D}=0.9$, with a strong thick cold boundary layer at the top and a weak hot boundary layer at the bottom. 


$$
R a_{s a}=2 \times 10^{4}
$$$$
R a_{s a}=4 \times 10^{4}
$$

Full compressible
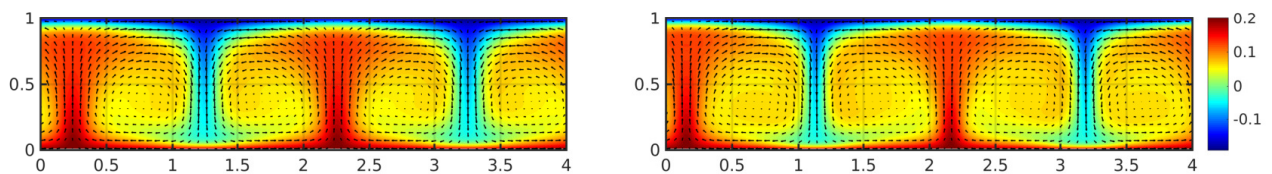

Anelastic Aproximation
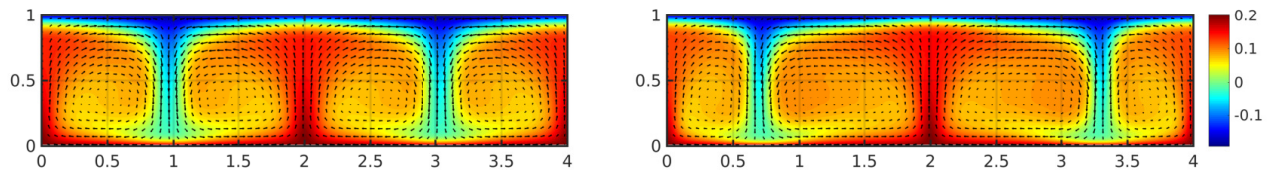

Anelastic liquid approximation
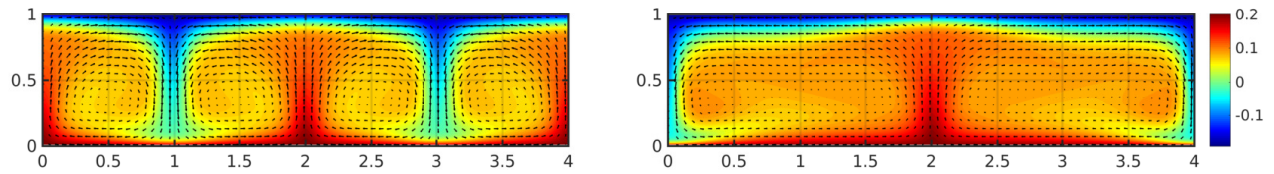

Figure 4. Comparison of the structure of the patterns of convection in full compressible FC, anelastic AA and anelastic liquid ALA approximations, for $r=3, \gamma=1.4$ and $\mathcal{D}=0.6(\epsilon=5 / 3)$. Black dots indicate the presence of two symmetricals convective cells, blue dot two asymmetrical cells and green dot a single cell.
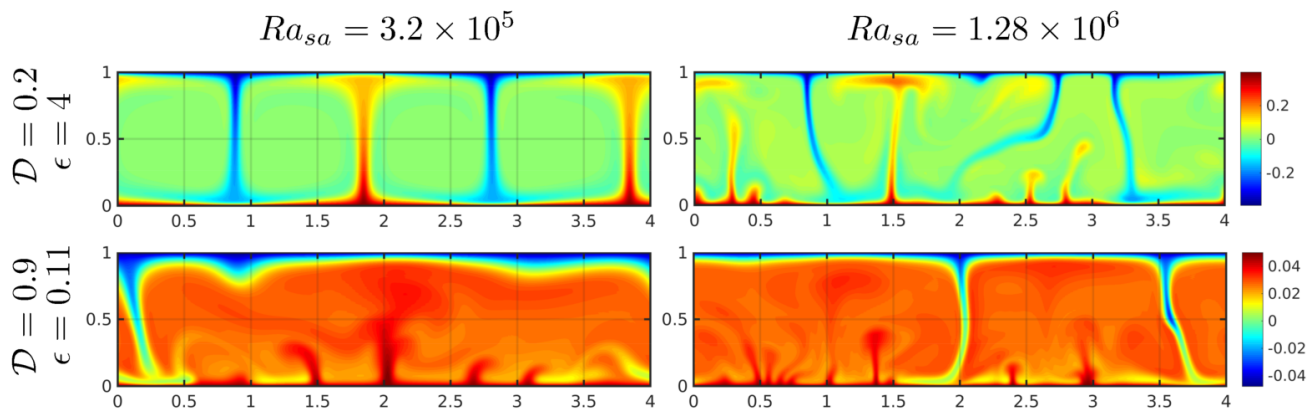

Figure 5. Structure of the plumes for the superadiabatic temperature $T_{s a}$ in the full compressible model for $r=3$ and $\gamma=1.4$

\section{Average temperature profiles}

A selection of time-averaged and horizontally-averaged temperature profiles are shown in Figs. 6 and 7. On Fig. 6, the average profiles are those obtained with the full compressible model $\mathrm{FC}$, for a heat capacity ratio equal to $\gamma=1.4$ and a temperature ratio $r=3$ between the bottom and top imposed temperatures. It can be observed that the profiles follow more or less the adiabatic reference profile in the bulk of the cavity with boundary layers where the temperature profiles bend to match the boundary 

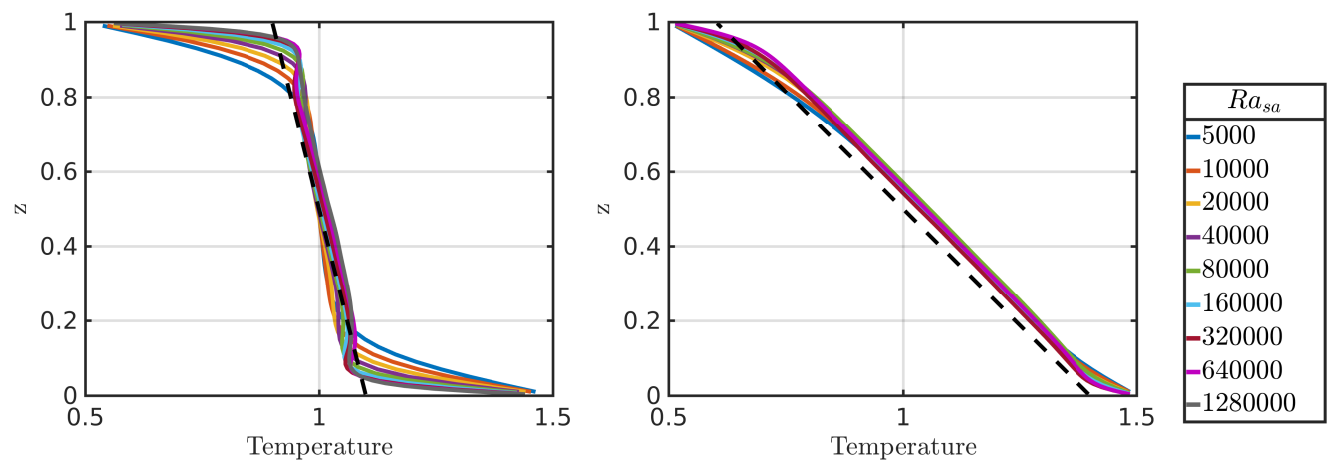

FiguRE 6. Average temperature profiles from the full compressible model FC, for $\gamma=1.4$ and a temperature ratio $r=3$. The value of the dissipation number is $\mathcal{D}=0.2(\epsilon=4)$ on the left-hand side and $\mathcal{D}=0.8(\epsilon=0.25)$ on the right-hand side. The profiles are shown for various values of the superadiabatic Rayleigh number (reference adiabatic profile shown as dashed line).
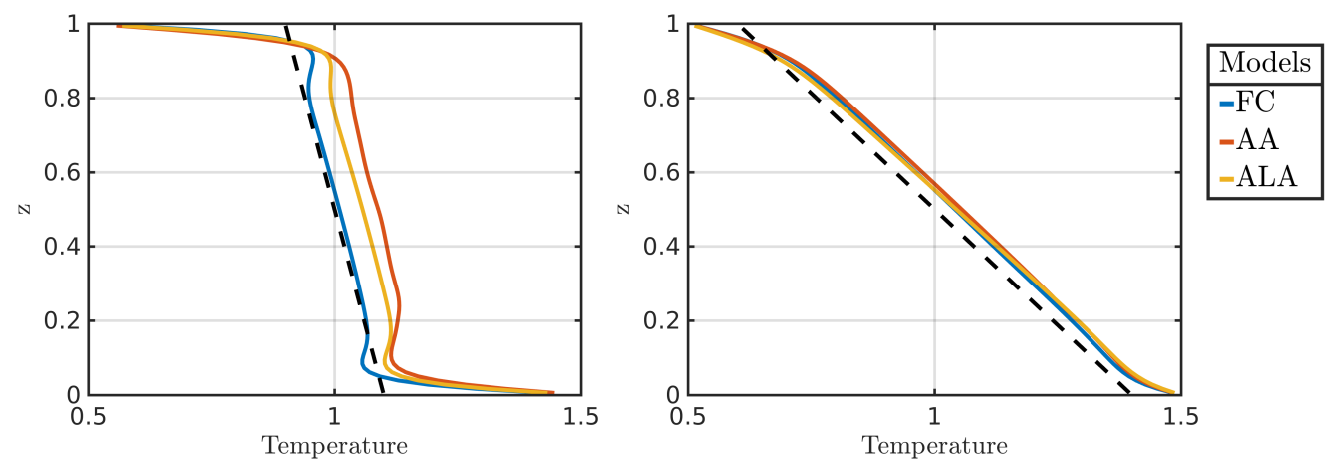

FiguRE 7. Average temperature profiles obtained from the full compressible FC, anelastic AA and anelastic liquid ALA models, for $R a_{s a}=320000, \gamma=1.4$ and $r=3$. The value of the dissipation number is $\mathcal{D}=0.2(\epsilon=4)$ on the left-hand side and $\mathcal{D}=0.8(\epsilon=0.25)$ on the right-hand side (reference adiabatic profile shown in dashed line).

conditions. As the superadiabatic Rayleigh number is increased, the thickness of the boundary layers is reduced. On the left-hand side, corresponding to a dissipation number $\mathcal{D}=0.2$, the profiles are all close to the reference adiabatic profile in the bulk of the fluid. It is reminded here that the adiabatic reference profile is such that the superadiabatic differences with the top and bottom temperatures are equal. On the contrary, on the right-hand side, corresponding to $\mathcal{D}=0.8$, the profiles follow a line parallel to, but distinct from, the adiabatic reference profile in the bulk of the flow. This means that the profiles still possess a temperature gradient in accordance to the adiabatic gradient, but that the actual profile is shifted by a constant temperature offset: a connected observation is that the temperature jump in the thermal boundary layers are not equal at the top and at the bottom since $Q_{t o p}=Q_{b o t}$ (see equation (5.6)). More often, the temperature jump across the upper boundary is larger than that across the lower boundary layer. This point will be discussed in section 8 .

In Fig. 7, the profiles obtained from different models (FC, AA and ALA) are shown for comparison. The superadiabatic Rayleigh number is equal to $320000, \gamma=1.4$ and $r=3$. Similarly to Fig. 6, the dissipation number is equal to 0.2 on the left-hand side and to 0.8 on the right-hand side. For $\mathcal{D}=0.2$, on the left-hand side, the three 


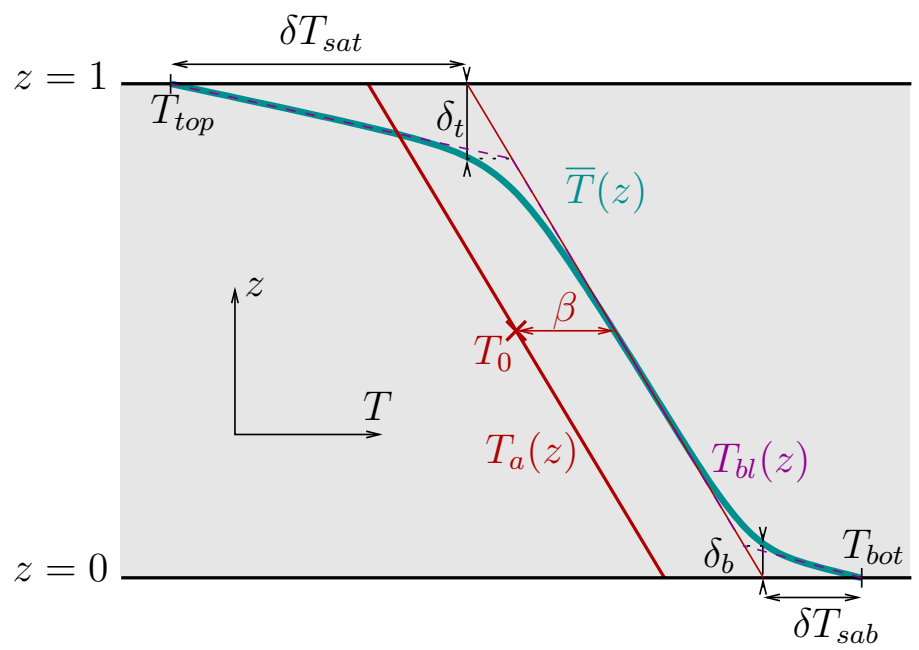

FIGURE 8. Typical horizontally-averaged temperature profile $\bar{T}(z)$, reference adiabatic profile $T_{a}(z)$, shifted adiabatic temperature profile $T_{a}(z)+\beta$ and boundary layer amplitudes $\left(\delta T_{s a b}\right.$ at the bottom, $\delta T_{\text {sat }}$ at the top), and thicknesses ( $\delta_{b}$ at the bottom, $\delta_{t}$ at the top). The dashed broken-line profile $T_{b l}(z)$ is an idealized piecewise linear profile, with linear core and linear boundary layers.

profiles corresponding to the full compressible FC model, anelastic AA and anelastic liquid ALA approximations, do not follow the same adiabat in the bulk of the flow. The full compressible profile has nearly no temperature offset compared to the adiabatic reference profile, while the anelastic approximation has a significant positive temperature offset, and the anelastic liquid a smaller offset. On the right-hand side, for $\mathcal{D}=0.8$, all three profiles follow the same adiabat, which is offset compared to the adiabatic reference profile. It is indeed expected that the anelastic approximations do a better job at reproducing the full compressible results when the dissipation number is large and close to its maximum $(\mathcal{D}=1$ for $r=3)$, because the superadiabatic temperature difference is small compared to the adiabatic temperature difference, thus reducing the uncertainty on the possible shift of the adiabatic profile.

Another feature of the average temperature profile is that it shows overshoots at the outer part of the boundary layers. This is more clearly visible in the case of $\mathcal{D}=0.2$ in Figs. 6 and 7. It can also be seen that the overshoots depend on the numerical model used (see Fig. 7): the anelastic profile AA has nearly no overshoot close to the top boundary layer while it is larger than its full compressible FC counterpart near the bottom boundary layer.

In order to understand analytically our numerical results, we consider a simplified representation of the average temperature profile, under the form of a piecewise linear function of $z$, one linear part in each boundary layer and a third one in the bulk of the fluid. This function, denoted $T_{b l}(z)$ (for 'boundary layers') is shown in Fig. 8. The bulk linear part follows the adiabatic gradient (slope $\mathcal{D}$ ), while the linear parts in the boundary layers are such that the total average heat flux is conducted along their gradient (slope $Q)$. This schematic profile captures indeed the typical temperature profiles of convecting compressible fluids whatever the approximations which are used (see Fig. 6 or 7 ) with the exceptions of the slight temperature overshoots that occur at the transitions between the conductive boundary layers and the adiabatic bulk. The profile must be anchored at the boundary conditions on temperatures (2.18) set by the ratio $r$. The last free parameter is 
the temperature offset $\beta$ of the adiabat in the core of the fluid. The analytical expression of this empirical profile is as follows

$$
\begin{aligned}
0<z<\delta_{b} & T_{b l}(z)=1+\frac{\delta T}{2}-z Q, \\
\delta_{b}<z<1-\delta_{t} & T_{b l}(z)=1+\beta-\mathcal{D}(z-1 / 2), \\
1-\delta_{t}<z<1 & T_{b l}(z)=1-\frac{\delta T}{2}-Q(z-1),
\end{aligned}
$$

where $\delta T=2(r-1) /(r+1)$. The expressions for the thickness of the boundary layers are obtained by continuity of the temperature profile

$$
\delta_{b}=\frac{(\delta T-\mathcal{D}) / 2-\beta}{Q-\mathcal{D}}, \quad \delta_{t}=\frac{(\delta T-\mathcal{D}) / 2+\beta}{Q-\mathcal{D}} .
$$

Those expressions can be further simplified when using the notations $\delta T_{s a}=\delta T-\mathcal{D}$ and $Q_{s a}=Q-\mathcal{D}$, for the total superadiabatic temperature difference and superadiabatic heat flux respectively. The total superadiabatic temperature difference $\delta T_{s a}$ is the sum of the superadiabatic temperature differences occurring across the bottom and the top boundary layers $\delta T_{s a}=\delta T_{s a b}+\delta T_{\text {sat }}$

$$
\delta_{b}=\frac{\delta T_{s a} / 2-\beta}{Q_{s a}}, \quad \delta_{t}=\frac{\delta T_{s a} / 2+\beta}{Q_{s a}} .
$$

We will see in section 8 that various numerical results will be recovered from this simple profile when we will derive an analytical expression of the offset $\beta$ as a function of the parameters $r$ and $\mathcal{D}$.

\section{An empirical rationale}

Let us consider the general asymmetry of the temperature profile. We attempt here to derive a rationale to explain the relative difference between the top and bottom boundary layer thicknesses. We shall then infer the consequences of this asymmetry on dissipation, entropy sources and heat flux. The ratio of the boundary layer thicknesses $x=\delta_{t} / \delta_{b}$ is also equal to the ratio of superadiabatic temperature jumps $x=\delta T_{\text {sat }} / \delta T_{\text {sab }}$ (because the thermal conductivity is uniform, see Fig. 8) and takes the following expression

$$
x=\frac{\delta_{t}}{\delta_{b}}=\frac{\delta T_{\text {sat }}}{\delta T_{\text {sab }}}=\frac{(\delta T-\mathcal{D}) / 2+\beta}{(\delta T-\mathcal{D}) / 2-\beta},
$$

We have determined the average temperature profile of our numerical simulations and found the closest adiabat followed in the bulk of the fluid. This provides both the offset $\beta$ and the ratio $x$ of the thicknesses of top to bottom boundary layers. This value is plotted in Fig. 9 as a function of the dissipation number for different sets of parameters and different approximations. Contrary to the incompressible finite Prandtl experiments described in $\mathrm{Wu} \&$ Libchaber (1991), the values of the ratio $x$ are mainly larger than unity. As we shall see below, the relative thickness of the boundary layers might be due to the relative thermal diffusivity, hence due to density in our simulations with uniform thermal conductivity (and heat capacity $c_{p 0}$ for ideal gases). In the paper of $\mathrm{Wu}$ and Libchaber, density was affected by temperature only, hot at the bottom, cold at the top, producing a lower density at the bottom than at the top. In our case, pressure effects dominate (except for small values of $\mathcal{D}$ ): large pressure at the bottom, low pressure at the top leads to a larger density at the bottom than at the top.

Now, we are going to use an empirical rule - a rationale - along with the piecewise 


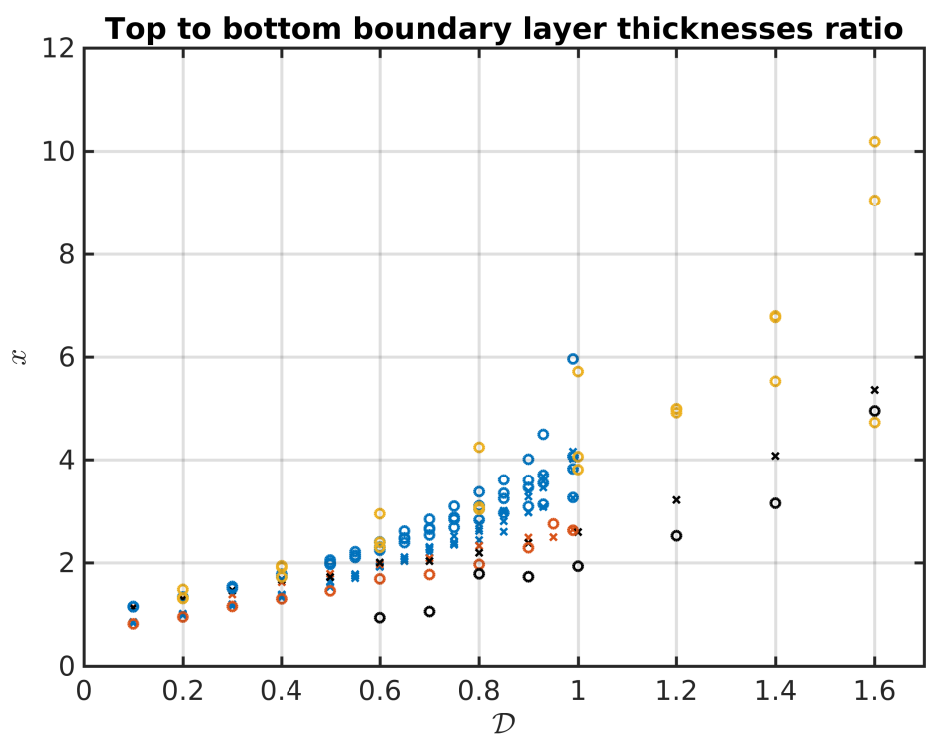

FIGURE 9 . The ratio of boundary layers thicknesses $x$ is plotted against the dissipation number: crosses $\times$ correspond to full-compressible calculations, circles $\circ$ to anelastic modelling. Blue points correspond to $r=3, \gamma=1.4$ and different superadiabatic Rayleigh numbers, brown points to $r=3, \gamma=5 / 3$ and $R a_{s a}=320000$, black points to $r=10, \gamma=5 / 3$ and $R a_{s a}=320000$, finally yellow points correspond to $r=10, \gamma=1.4, R a_{s a}=10^{4}, R a_{s a}=10^{5}$ and $R a_{s a}=10^{6}$.

linear profile defined above, that will enable us to model some of our numerical results. In fact, we are going first to test three different rationales against the numerical results and select the most satisfactory. This is inspired by the work of Wu \& Libchaber (1991), where they also tested different hypotheses against experimental results in the context of non-Boussinesq, nearly incompressible Rayleigh-Bénard convection.

The first hypothesis consists in assuming that the Rayleigh number of each boundary layer is critical: for our purpose, we simply assume that they take always the same value. The second hypothesis is to be found in Wu \& Libchaber (1991)'s paper (where they also tested the constant Rayleigh hypothesis) and consists in assuming that the top and bottom potential temperature differences are equal: the potential temperature difference is such that it makes the Rayleigh number critical. Finally, we introduce a third rationale, consisting in assuming that the timescale of the development of the boundary layers is identical at the top and bottom.

The ratio of top, $R a_{t}$, to bottom, $R a_{b}$, boundary layer Rayleigh numbers is now evaluated considering that both thermal conductivity and viscosity are uniform

$$
\frac{R a_{t}}{R a_{b}}=\frac{\alpha_{t} \rho_{t}^{2} \delta T_{s a t} \delta_{t}^{3}}{\alpha_{b} \rho_{b}^{2} \delta T_{s a b} \delta_{b}^{3}}=\left(\frac{P_{t}}{P_{b}}\right)^{2}\left(\frac{T_{b}}{T_{t}}\right)^{3} x^{4},
$$

where all values evaluated at the top have a subscript $t$ and those at the bottom a subscript $b$. As we want to compare different rationales, we also make the choice to compare quantities simply proportional to $x$, so that we shall take the expression $(8.2)$ to the power one fourth. Hence, the following function $R 1$ corresponds to the ratio of the Rayleigh numbers to the power one fourth

$$
R 1=\left(\frac{P_{t}}{P_{b}}\right)^{1 / 2}\left(\frac{T_{b}}{T_{t}}\right)^{3 / 4} x .
$$


From our numerical calculations, if we can define the top and bottom pressures and temperatures, which we discuss later, and evaluate $x$ by fitting the average temperature profile with the closest adiabat line, we can then compute $R 1$ and values close to unity would indicate that the first rationale is a good representation of the simulations and values departing from 1 that the Rayleigh numbers are not equal in both boundary layers.

We follow a similar path for the second rationale. The ratio of potential temperature differences can be written

$$
\frac{\theta_{b}}{\theta_{t}}=\frac{\alpha_{t} \rho_{t}^{2} \delta_{t}^{3}}{\alpha_{b} \rho_{b}^{2} \delta_{b}^{3}}=\left(\frac{P_{t}}{P_{b}}\right)^{2}\left(\frac{T_{b}}{T_{t}}\right)^{3} x^{3},
$$

leading to the following function $R 2$ for the second rationale

$$
R 2=\left(\frac{P_{t}}{P_{b}}\right)^{2 / 3} \frac{T_{b}}{T_{t}} x .
$$

If those ratios are close to unity, then potential temperature differences for both boundary layers are indeed nearly equal.

In the third rationale, we consider that the top and bottom boundary layers develop over the same time-scale $\tau$. So that their typical diffusion thickness $\delta \sim \sqrt{\kappa \tau}$ (where $\kappa$ is the thermal diffusivity) depends explicitly on density $\delta \sim \sqrt{k /\left(\rho c_{p}\right) \tau}$. Hence the ratio of boundary layer thicknesses is inversely proportional to the square root of the density ratio. From this point, we can build the function $R 3$ corresponding to this rationale

$$
R 3=\left(\frac{\rho_{t}}{\rho_{b}}\right)^{1 / 2} x=\left(\frac{P_{t}}{P_{b}}\right)^{1 / 2}\left(\frac{T_{b}}{T_{t}}\right)^{1 / 2} x .
$$

Again, values close to unity for these functions is a validation of the third rationale, based on a constant development duration for the thermal boundary layers.

Like Wu \& Libchaber (1991), we consider the average within a boundary layer when we need to evaluate a physical property relative to that boundary layer. However, the full compressible case FC and an anelastic model (AA or ALA) are treated differently: for anelastic models, all quantities are evaluated on the adiabatic reference profile, so that physical quantities in a particular boundary layer must be evaluated at the conditions of the adiabatic reference profile using (3.5) and (3.7). In addition, we consider their value at the boundaries, which is only very slightly different from their value in the middle of the thin boundary layers so that, for the AA and ALA models, we can consider that

$$
\begin{aligned}
T_{t} & =1-\mathcal{D} / 2 \\
T_{b} & =1+\mathcal{D} / 2 \\
P_{t / b} & =\frac{\mathcal{D}}{T_{b}^{\frac{\gamma}{\gamma-1}}-T_{t}^{\frac{\gamma}{\gamma-1}}} T_{t / b}^{\frac{\gamma}{\gamma-1}} .
\end{aligned}
$$

From our anelastic computations and using those expressions for the top and bottom pressures and temperature we can can therefore evaluate the $R 1_{A}, R 2_{A}$ and $R 3_{A}$ following (8.3), (8.5) and (8.6), where we add the subscript $A$ for anelastic.

In the case of full compressible results FC, we take into account the thermal boundary layers to evaluate temperatures $T_{b}$ and $T_{t}$, but we can safely approximate the exact pressure by the pressure along the adiabat. In addition, we now need to take the offset $\beta$ 

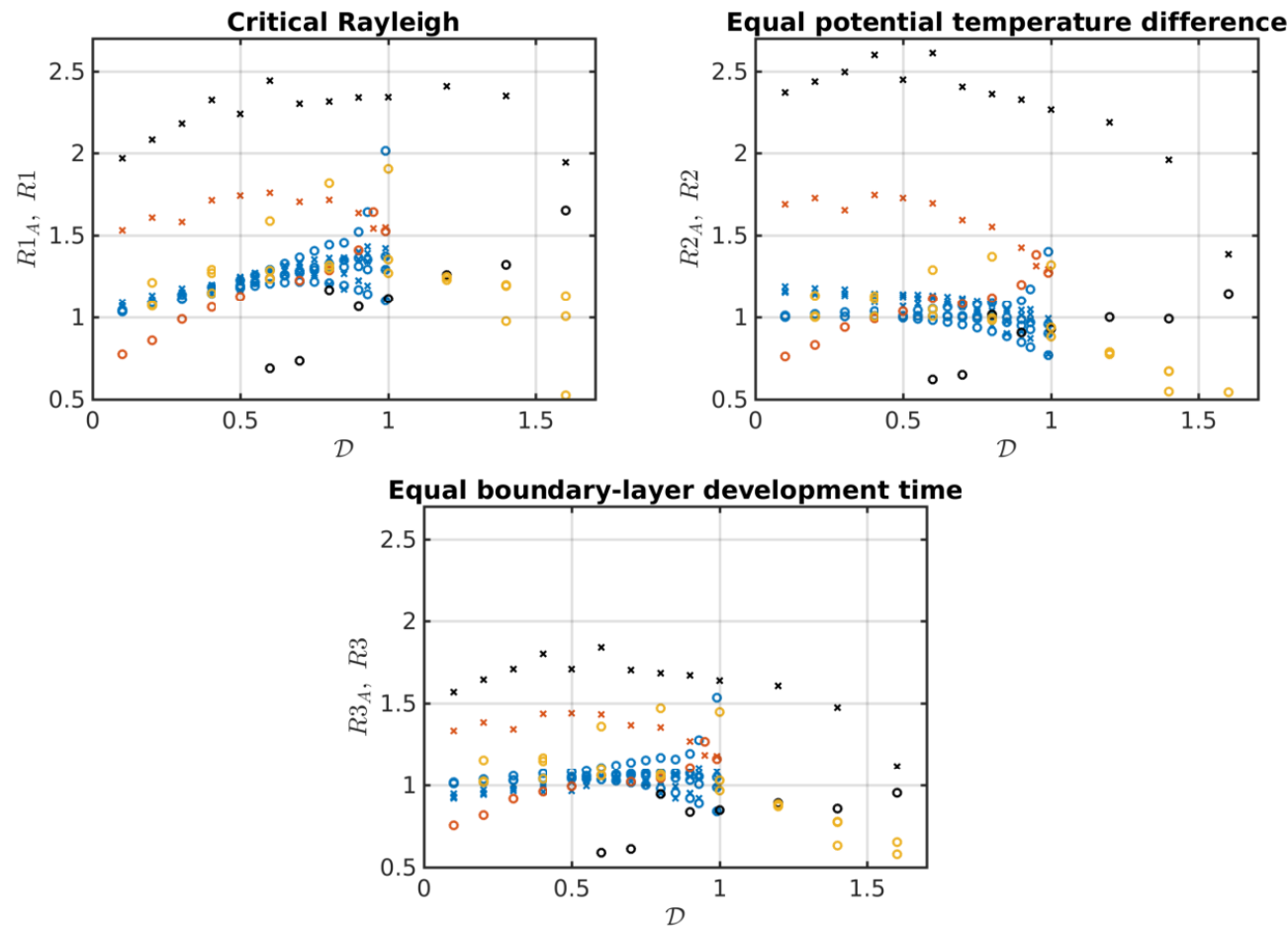

FiguRE 10. The three quantities corresponding to the three different rationales are plotted against the dissipation number: crosses $\times$ correspond to full-compressible calculations, circles $\circ$ to anelastic modelling. Blue points correspond to $r=3, \gamma=1.4$ and different superadiabatic Rayleigh numbers, brown points to $r=3, \gamma=5 / 3$ and $R a_{s a}=320000$, black points to $r=10$, $\gamma=5 / 3$ and $R a_{s a}=320000$, finally yellow points correspond to $r=10, \gamma=1.4, R a_{s a}=10^{4}$, $R a_{s a}=10^{5}$ and $R a_{s a}=10^{6}$.

into account. Instead of the anelastic estimates (8.7), for the FC case, we must now use

$$
\begin{aligned}
T_{t} & =\frac{1}{2}\left(1+\beta-\mathcal{D} / 2+\frac{2}{r+1}\right) \\
T_{b} & =\frac{1}{2}\left(1+\beta+\mathcal{D} / 2+\frac{2 r}{r+1}\right) \\
P_{t} & =\frac{\mathcal{D}}{(1+\beta+\mathcal{D} / 2)^{\frac{\gamma}{\gamma-1}}-(1+\beta-\mathcal{D} / 2)^{\frac{\gamma}{\gamma-1}}}(1+\beta-\mathcal{D} / 2)^{\frac{\gamma}{\gamma-1}} \\
P_{b} & =\frac{\mathcal{D}}{(1+\beta+\mathcal{D} / 2)^{\frac{\gamma}{\gamma-1}}-(1+\beta-\mathcal{D} / 2)^{\frac{\gamma}{\gamma-1}}}(1+\beta+\mathcal{D} / 2)^{\frac{\gamma}{\gamma-1}} .
\end{aligned}
$$

where $\beta$ is obtained from $x$ by inverting equation (8.1)

$$
\beta=\frac{\delta T-\mathcal{D}}{2} \frac{x-1}{x+1}
$$

The pressure expressions are computed along the adiabat taking into account the appropriate $\beta$-offset of the temperature (in agreement with (3.7)).

All functions $R 1, R 1_{A}, R 2, R 2_{A}, R 3$ and $R 3_{A}$ are plotted in Fig. 10. It can be seen that the rationale 3 is slightly better than 2 or 1: all points (AA and FC) are closer to unity in the range of our numerical simulations. Hence, in the following, we are going to 
use the third rationale, based on a constant timescale of boundary layer development, i.e. deduce $x$ from

$$
x=\left(\frac{\rho_{b}}{\rho_{t}}\right)^{1 / 2}=\left(\frac{P_{b}}{P_{t}}\right)^{1 / 2}\left(\frac{T_{t}}{T_{b}}\right)^{1 / 2}
$$

in the anelastic and full compressible cases using (8.7) or (8.8), to derive conclusions regarding the dependency of the heat flux and viscous dissipation versus the dissipation number. In the anelastic case, the expression (8.10) provides $x$ explicitly in terms of $\mathcal{D}$ and $\gamma$, however this is not the case for the full compressible expression due to the presence of $\beta$ which is itself linked to $x$ by equation (8.9): one can use an iterative numerical method in order to obtain finally $x$ as a function of $\mathcal{D}, \gamma$ and $r$.

The dependency of the Nusselt number versus the dissipation number (the numerical results are found in Figs. 12 and 13) can be derived from our rationale. Because the isentropic (or adiabatic) profile represents an equilibrium state of the fluid in terms of buoyancy, we decompose the total heat flux in a flux conducted along the adiabat, $Q_{a}=$ $\Delta T_{a}=\mathcal{D}$, and a so-called superadiabatic heat flux, $Q_{s a}=Q-Q_{a}$. The superadiabatic heat flux is driven by the superadiabatic temperature difference $\Delta T-\Delta T_{a}$ and we introduce a superadiabatic Nusselt number defined as the ratio of the superadiabatic heat flux to the flux conducted by the superadiabatic temperature difference:

$$
N u_{s a}=\frac{Q_{s a}}{\Delta T_{s a}},
$$

From Fig. 8, the dimensionless superadiabatic heat flux $Q_{s a}$ can be written

$$
Q_{s a}=\frac{\delta T_{s a t}}{\delta_{t}}
$$

Using the definition of $x$, from equation (8.1), and expressing the total dimensionless superadiabatic temperature difference $\delta T_{s a}=\delta T_{\text {sat }}+\delta T_{\text {sab }}$, we have

$$
\delta T_{\text {sat }}=\frac{x}{1+x} \delta T_{s a} .
$$

Furthermore, we adopt an extended version of our rationale. We now assume that the timescale for the development of top and bottom boundary layers depends on the superadiabatic Rayleigh number only (and not on the dissipation number $\mathcal{D}$, nor $\gamma$, nor $r)$. This implies that the boundary layer thickness is inversely proportional to the square root of density, so that equations (8.12) and (8.13) lead to

$$
N u_{s a}=\frac{Q_{s a}}{\delta T_{s a}}=2 \frac{x}{1+x} \sqrt{\rho_{t}} N u_{0},
$$

where $N u_{0}$ is the Nusselt number of the anelastic calculation AA at small dissipation number $\mathcal{D} \sim 0$ and same Rayleigh number (for which density is unity everywhere, including in the top boundary layer). This last expression can be made symmetrical since the superadiabatic heat flux can be expressed in terms of the bottom parameters $Q_{s a}=\delta T_{s a b} / \delta_{b}$. This would lead to

$$
N u_{s a}=2 \frac{1}{1+x} \sqrt{\rho_{b}} N u_{0} .
$$

Taking the geometric average of (8.14) and (8.15) and inserting the definition of $x$ (see (8.10)), gives the following symmetrical expression:

$$
N u_{s a}=2 \frac{\sqrt{\rho_{b} \rho_{t}}}{\sqrt{\rho_{b}}+\sqrt{\rho_{t}}} N u_{0}=\frac{2}{\sqrt{1-\gamma^{-1}}} \frac{\sqrt{P_{b} P_{t}}}{\sqrt{P_{b} T_{t}}+\sqrt{P_{t} T_{b}}} N u_{0} .
$$




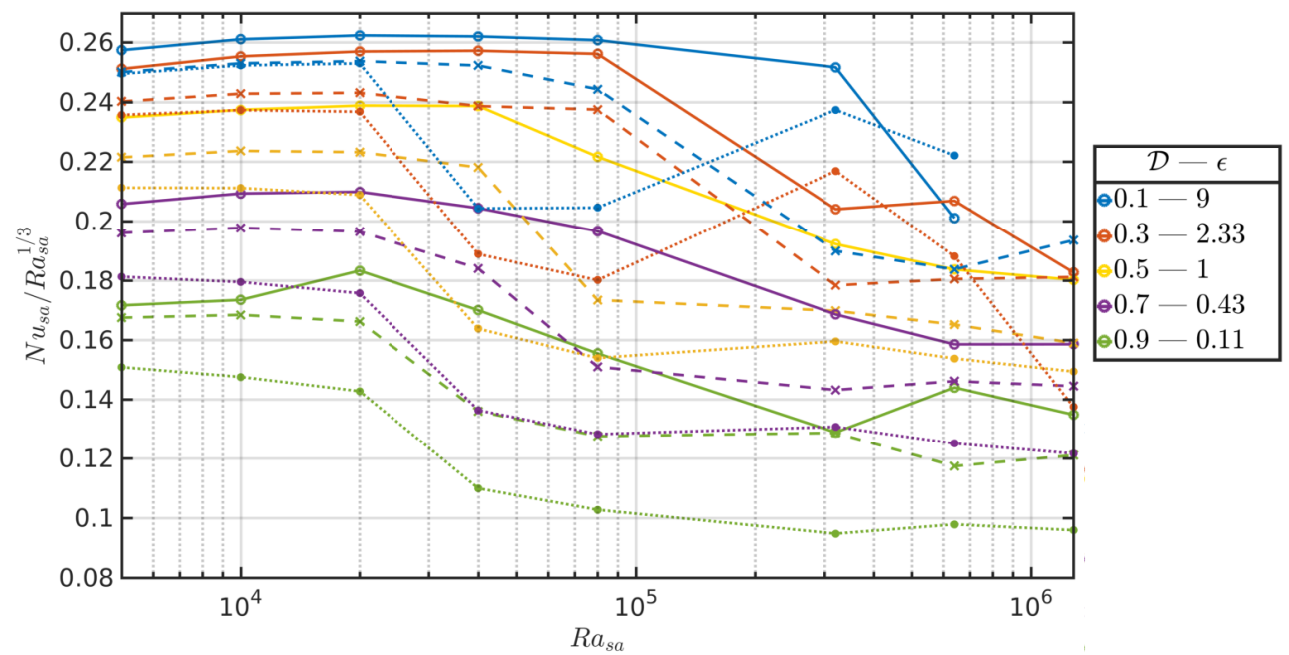

FIGURE 11. Superadiabatic Nusselt number $N u_{s a}=Q_{s a} / \Delta T_{s a}$, divided by $R a_{s a}^{1 / 3}$, as a function of $R a_{s a}$ for full compressible calculations (solid lines), anelastic approximation (dashed lines) and anelastic liquid approximation (dotted lines), for $r=3, \gamma=1.4$ and a range of values of the dissipation number $\mathcal{D}$.

Now, the expression above (8.16) is evaluated differently for anelastic calculations $N u_{s a A A}$ (where pressures and temperatures are given by (8.7)) and for fully compressible calculations $N u_{s a F C}$ for which we use (8.8). We will see in section 9 that the rationale describes indeed correctly the variation of the Nusselt number with the dissipation number.

\section{Heat flux}

We now analyse the outcome of the numerical calculations in terms of global heat flux transferred through the layer. We have run calculations for a temperature ratio $r=3$ and some others for $r=10$. The ratio of heat capacities has been set to $\gamma=1.4$, corresponding to diatomic gases. Ranges of the other two available dimensionless numbers, $\mathcal{D}$ and $R a_{s a}$ have been investigated. Convection can only occur when the conductive gradient $a=2(r-1) /(r+1)$ corresponding to the difference of the imposed temperatures $(2.18)$ is larger than the adiabatic gradient $\mathcal{D}$. Equivalently, for a given value of $r$, the value of $\mathcal{D}$ must lie within the interval $\left[0 ; \mathcal{D}_{\max }\right]$, where $\mathcal{D}_{\max }$ has been defined in equation (2.22). For $r=3$ the dissipation number $\mathcal{D}$ is comprised in $[0 ; 1]$. For $r=10$, this interval is $[0 ; 18 / 11]$. When $\mathcal{D}$ exceeds $\mathcal{D}_{\max }$, the fluid is stably stratified and no convection can develop. The range of $R a_{s a}$ is limited by resolution and calculation time.

\subsection{Full compressible $F C$ versus Anelastic approximation AA results}

The value of the heat flux is compared between the full compressible calculations and the corresponding anelastic approximation. For convection at infinite $\operatorname{Pr}$ number, it is believed that $N u_{s a} \propto R a_{s a}^{1 / 3}$ in the Boussinesq approximation (Malkus 1954; Grossmann \& Lohse 2000) and we shall plot our compressible results relative to the same scaling. In Fig. 11, we plot the superadiabatic Nusselt number scaled with $R a_{s a}^{1 / 3}$, for different values of the dissipation number $\mathcal{D}$. The results for the full compressible and anelastic 


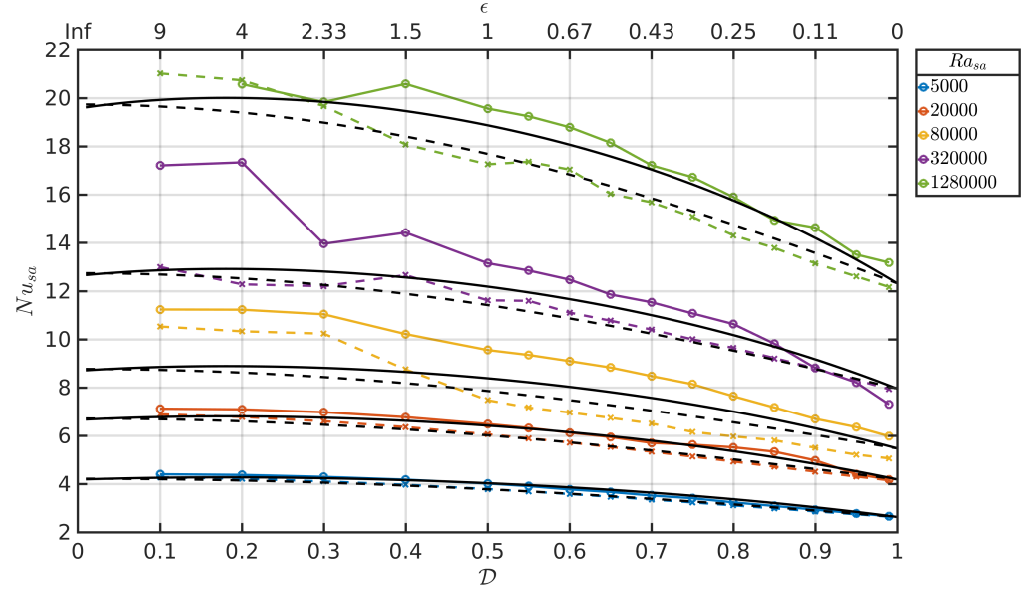

FiguRE 12. Superadiabatic Nusselt number $N u_{s a}=Q_{s a} / \Delta T_{s a}$ as a function of $\mathcal{D}$ for full compressible calculations (solid lines) and anelastic approximation (dashed lines), for $r=3$, $\gamma=1.4$ and a range of values of the superadiabatic Rayleigh number $R a_{s a}$. The black lines correspond to the "rationale" expression (8.16) derived in section 8 for the full-compressible case (full lines (8.8)) and anelastic approximation (dashed lines (8.7)). The free parameter $N u_{0}$ is chosen such that the anelastic profile has the best fit with the anelastic numerical results.

approximation are plotted together, for an imposed temperature ratio $r=3$. The scaling $N u_{s a} \sim R a_{s a}^{1 / 3}$ is roughly correct for all values of $\mathcal{D}$, up to the maximum $\mathcal{D}=1$ for $r=3$. More precisely, we identify a transition, near $R a_{s a}=10^{5}$, with a decrease of the prefactor of $25 \%$ in the $R a_{s a}^{1 / 3}$ scaling. This corresponds to the change from a steady to an unsteady convection regime (see below). This transition, observed here in $2 \mathrm{D}$ calculations, is likely to occur for lower Rayleigh numbers in 3D calculations. The same results of superadiabatic Nusselt numbers are plotted in Fig. 12, as a function of $\mathcal{D}$, for several values of the superadiabatic Rayleigh number $R a_{s a}$. The values of $N u_{s a}$ are constant at small $\mathcal{D}$ and decline for larger $\mathcal{D}$ by approximately $40 \%$ when $\mathcal{D}=1$. The effect of the dissipation number seems to be independent of the superadiabatic Rayleigh number. We also plot in Figs. 11 and 12 the predictions (8.16) for full-compressible (using 8.8) and for the anelastic approximation (using 8.7), obtained from the heuristic reasoning developed in section 8 , based on the relative development of the top and bottom boundary layers. The numerical Nusselt numbers follow quite well the predictions of this rationale. The values of $N u_{s a}$ for $r=10$ has a similar behavior as can be seen in Fig. 13 where the $N u_{s a}$ is represented as a function of $\mathcal{D}$ up to the maximum $\mathcal{D}=2(r-1) /(r+1)$ for the anelastic calculations.

On both Figs. 11 and 12, we can observe that the Nusselt numbers computed with the full compressible method are larger than those computed using the anelastic approximation. This is made clear in Fig. 14 on which we represent the relative difference in the superadiabatic Nusselt numbers $\left(N u_{s a}^{F C}-N u_{s a}^{A A}\right) / N u_{s a}^{F C}$ on a color scale in the plane $\mathcal{D}-R a_{s a}$, where $N u_{s a}^{F C}$ is the superadiabatic Nusselt number obtained with the full compressible method, while $N u_{s a}^{A A}$ is obtained using the anelastic approximation. Nearly all values are positive (red color) and indicate that the heat flow is 10-20\% larger than what is obtained by the anelastic approximation. Moreover, we also indicate the points in this parameter space $\mathcal{D}-R a_{s a}$ where numerical calculations have been performed. Full circles indicate a regime of steady convection, empty circles an unsteady regime, for full compressible results FC and anelastic results AA, left and right symbols respectively. 


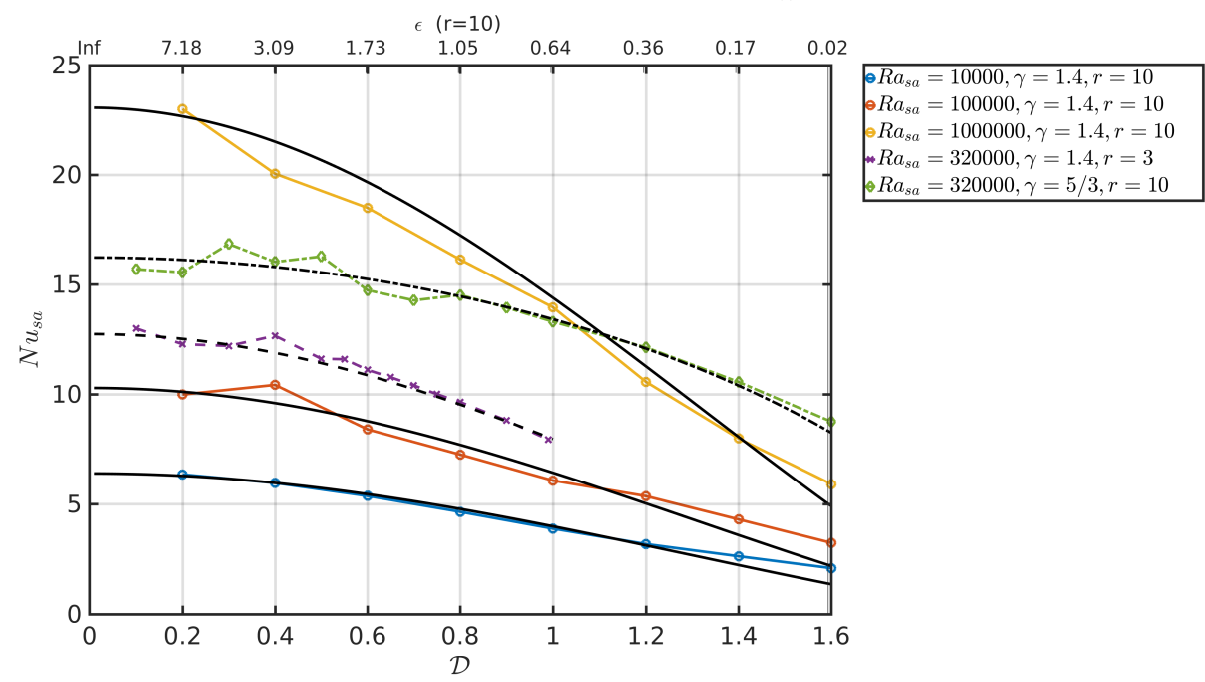

FIgURE 13. Superadiabatic Nusselt number $N u_{s a}=Q_{s a} / \Delta T_{s a}$ as a function of $\mathcal{D}$ for the anelastic approximation with different values of $\gamma$ and a range of values of the superadiabatic Rayleigh number $R a_{s a}$. Black curves correspond to the analytic expression (8.16), using (8.7).

The color of the points depends on the spatial structure of convection as in Fig. 4: two symmetric rolls (black), two asymmetric rolls (blue), one large convection roll (green), or indefinite (gray). A region of maximum relative difference (intense red, positive) of the Nusselt number corresponds to the line $R a=8 \times 10^{4}$, just below $10^{5}$. It basically corresponds to the region where the anelastic results have already undergone transition to unsteady convective rolls (empty symbols), while full compressible results are still steady (full symbols). This is also visible in Fig. 11 where the transition to a smaller prefactor occurs at a smaller Rayleigh number for anelastic solutions compared to full compressible solutions. That transition is dependent on the $2 \mathrm{D}$ nature of the simulations and would likely occur at small Rayleigh numbers in 3D. Conversely, regions where the relative difference is small or slightly negative (white background or slightly yellow) corresponds to regions of the parameter space where the structure of convection is similar between full compressible and anelastic results.

Let us now consider the specific question of the convergence of the anelastic AA to the full compressible FC results in terms of heat fluxes. This convergence is expected when $\epsilon$ is very small, or equivalently when the dissipation parameter approaches its maximum value (see equations $(2.22),(2.23)$ and $(2.24)$ ). It can be seen in Fig. 14, that the red color has a tendency to fade out at small epsilon, so that the relative difference of the heat fluxes between anelastic AA and full compressible FC results is rather small near the maximum value of $\mathcal{D}\left(\mathcal{D}_{\max }=1\right.$ for $\left.r=3\right)$. This is particularly clear at small values of the superadiabatic Rayleigh number but is obscured between $R a_{s a} \sim 4 \times 10^{4}$ and $R a_{s a} \sim 8 \times 10^{4}$ : our understanding is that the transition from two to one rolls of convection depends on very small details of the mathematical model and may require a tiny value of $\epsilon$ to be visible. This convergence can be seen again to a lesser extent at larger values of $R a_{s a}$. However, we will see in section 11 about the entropy balance, that the convergence of AA results towards FC results necessitates smaller and smaller values of $\epsilon$ as the superadiabatic Rayleigh number $R a_{s a}$ becomes larger and larger. 


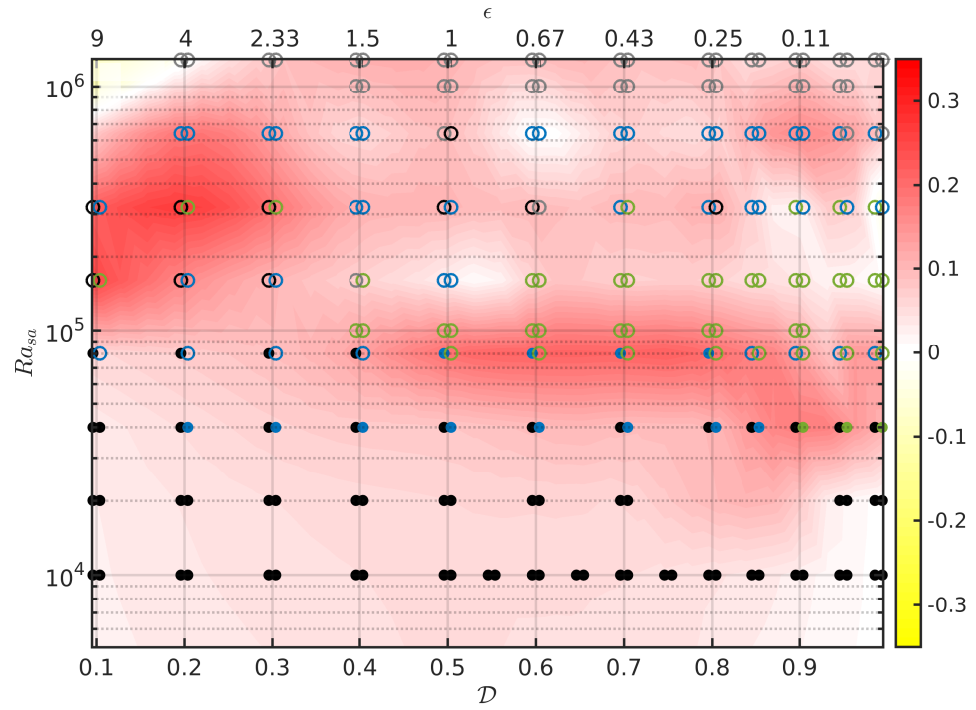

FiguRE 14. The color scale shows the relative difference $\left(N u_{s a}^{F C}-N u_{s a}^{A A}\right) / N u_{s a}^{F C}$ of the superadiabatic Nusselt number $N u_{s a}=Q_{s a} / \Delta T_{s a}$ as a function of $\mathcal{D}$ and $R a_{s a}$ between full compressible FC and anelastic AA calculations, for $r=3, \gamma=1.4$. The circles indicate the actual values of the parameters where a numerical solution has been computed, full-compressible FC or anelastic AA corresponding to the symbol on the left-hand side and on the right-hand side, respectively. The circles denote unsteady statistically stationary solutions (open symbols) or steady solutions (full symbols). The color of the circles show two symmetric convection rolls (black), two asymmetric rolls (blue) or one convection roll (green), while gray symbols correspond to a not well defined geometry of the convection (see Fig. 4 for examples).

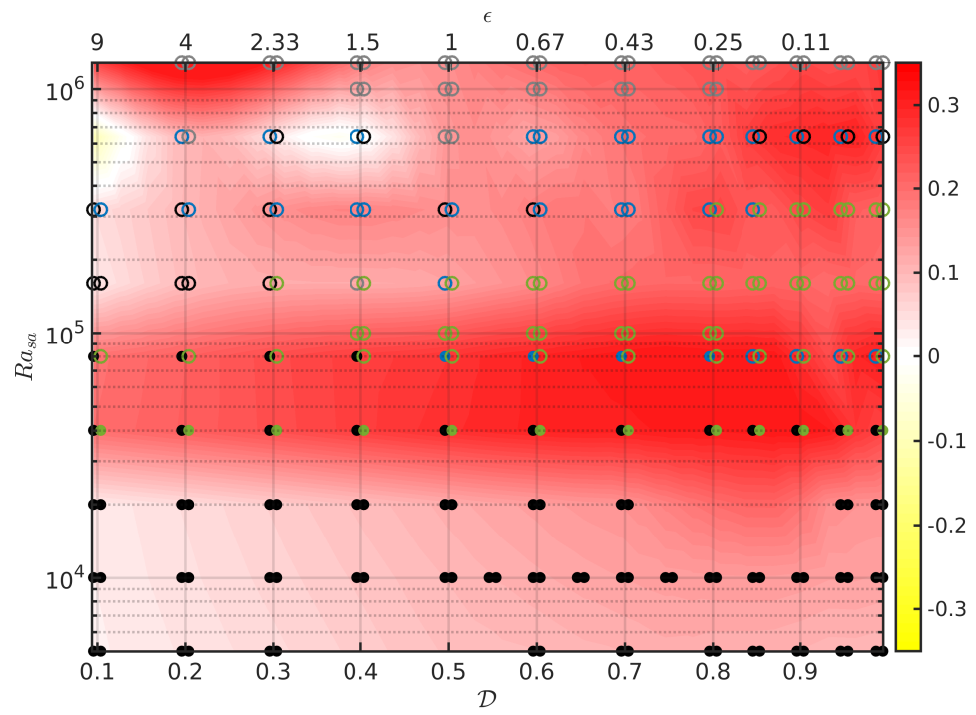

FIGURE 15. Relative difference $\left(N u_{s a}^{F C}-N u_{s a}^{A L A}\right) / N u_{s a}^{F C}$ of the superadiabatic Nusselt number $N u_{s a}=Q_{s a} / \Delta T_{s a}$ as a function of $\mathcal{D}$ and $R a_{s a}$ between full compressible $\mathrm{FC}$ and anelastic liquid ALA calculations, for $r=3, \gamma=1.4$. The points (colored circles) follow the same code as in Fig. 14, except they correspond now to the full compressible (FC, left symbols) and anelastic liquid (ALA, right symbols) numerical calculations. 


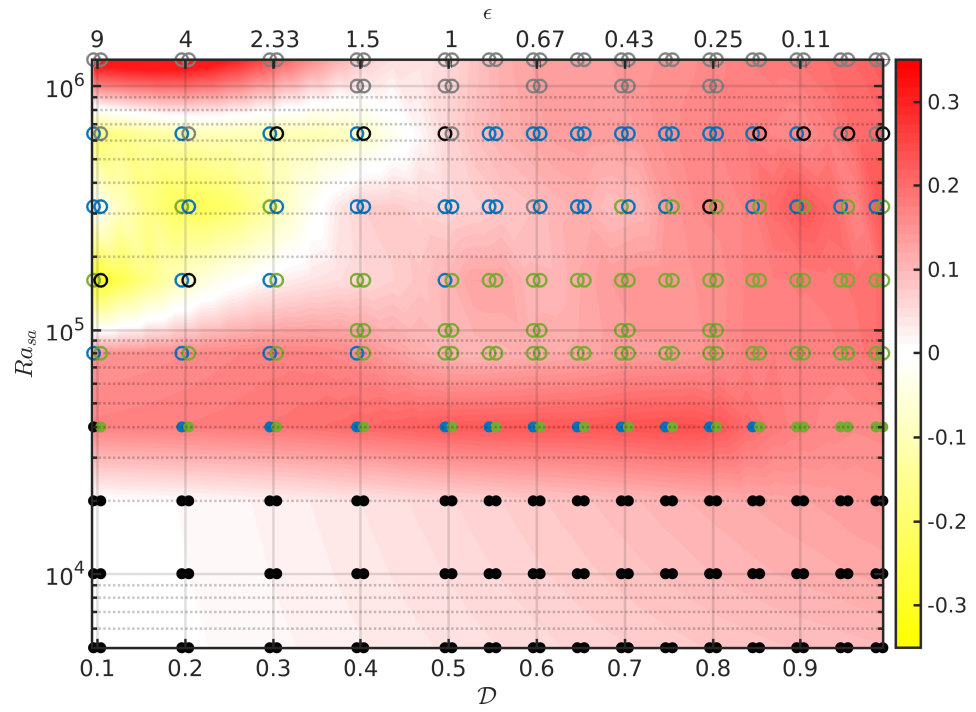

FIgURE 16. Relative difference $\left(N u_{s a}^{A A}-N u_{s a}^{A L A}\right) / N u_{s a}^{A A}$ of the superadiabatic Nusselt number $N u_{s a}=Q_{s a} / \Delta T_{s a}$ as a function of $\mathcal{D}$ and $R a_{s a}$ between full anelastic AA and anelastic liquid ALA calculations, for $r=3, \gamma=1.4$. The points (colored circles) follow the same code as in Fig. 14, except they correspond now to the anelastic (AA, left symbols) and anelastic liquid (ALA, right symbols) numerical calculations.

\subsection{Full compressible FC versus Anelastic liquid approximation ALA results}

We now compare the full compressible FC and anelastic liquid approximation ALA results. Figure 15 shows the relative difference of the superadiabatic Nusselt numbers between the full compressible and anelastic liquid results. It is built similarly to Fig. 14 where full compressible results were compared to anelastic results. This color plot is also positive in the region of the parameter space we have investigated $(0<\mathcal{D}<1$ and $\left.5 \times 10^{3}<R a_{s a}<1.28 \times 10^{6}\right)$, with values even larger than in Fig. 14. The full compressible heat flux is always larger than the anelastic liquid heat flux, by about $30 \%$ when the supercritical Rayleigh number is above $2 \times 10^{4}$. Moreover, we can see a global trend, the relative difference increasing with $\mathcal{D}$ and possibly with $R a_{s a}$. Like in Fig. 14, there is also a region with enhanced positive relative difference along $R a=4 \times 10^{4}$ where the ALA approximation predicts unsteady convection before the FC case.

\subsection{Anelastic AA versus Anelastic liquid ALA approximation results}

Fig. 16 provides a comparison of the heat flux computed with the AA and ALA approximations. In most cases, the anelastic AA heat flux is larger than the corresponding anelastic liquid approximation ALA value. The effect of the early transition of ALA convection patterns with respect to the AA case is visible around $R a \sim 4 \times 10^{4}$, but in addition we can see a global trend of linear increase with $\mathcal{D}$ of the flux difference between AA and ALA results. This is consistent with the estimate provided by Anufriev et al. (2005), stating that the anelastic liquid approximation ALA should be valid when $\alpha T \mathcal{D}<<1$ (see their equation $(2.17 \mathrm{a})$ ).

\subsection{The compressible contribution to the heat flux}

Expressions (5.5) for the full compressible case and (5.6) for the anelastic case can be used to evaluate the contribution of heat transfer due to shear-stress power $(\mathcal{D} / R) \overline{v_{j} \tau_{z j}}$, 
in addition to the usual conduction term and enthalpy transport term. Integrating the heat flux over the vertical coordinate (unit dimensionless distance) leads to

$$
Q=\left\langle\rho h v_{z}\right\rangle-\frac{\mathcal{D}}{R}\left\langle v_{j} \tau_{z j}\right\rangle+\left(T_{b o t}-T_{t o p}\right) .
$$

Using the Newtonian rheology (2.5), integrating by parts, and using the boundary conditions, we can express the integrated flux as follows

$$
Q=\left\langle\rho h v_{z}\right\rangle+\frac{5}{3} \frac{\mathcal{D}}{R}\left\langle v_{z} \boldsymbol{\nabla} \cdot \mathbf{v}\right\rangle+\left(T_{b o t}-T_{\text {top }}\right) .
$$

It is expected that the shear-stress power contribution to the heat flux is positive: in general, when a parcel of fluid goes up $\left(v_{z}>0\right)$, it experiences an expansion $(\nabla \cdot \mathbf{v}>0)$, while conversely a descending fluid particle $\left(v_{z}<0\right)$ is associated with compression $(\nabla \cdot \mathbf{v}<0)$. However, this is not necessarily true for each fluid parcel: pressure fluctuations can locally cancel this effect, through a so-called 'buoyancy braking' effect (Hurlburt et al. 1984). Nevertheless, when an anelastic approximation is used, the integrated heat flux takes a simpler form, using the continuity equation $\nabla \cdot\left(\rho_{a} \mathbf{v}\right)=0$ :

$$
Q=\left\langle\rho h v_{z}\right\rangle-\frac{5}{3} \frac{\mathcal{D}}{R}\left\langle\frac{\mathrm{d} \ln \rho_{a}}{\mathrm{~d} z} v_{z}^{2}\right\rangle+\left(T_{b o t}-T_{\text {top }}\right) .
$$

Now, as $\mathrm{d} \ln \rho_{a} / \mathrm{d} z<0$, the shear-stress power contribution (middle term at the righthand side of $(9.3)$ ) to the integrated heat flux is necessarily positive everywhere. There is no classical result concerning the magnitude of the shear-stress power contribution to the heat flux. In the Boussinesq approximation, this contribution is exactly zero as can be seen from (9.2) because the divergence of the velocity field vanishes, or from (9.3) because the reference density $\rho_{a}$ is uniform. In the anelastic approximation, we show here that the magnitude of the shear-stress power contribution to the heat flux is quadratic in the dissipation parameter $\mathcal{D}$ near $\mathcal{D}=0$. For small values of $\mathcal{D}$, the velocity field $\mathbf{v}$ is nearly equal to its value at $\mathcal{D}=0$. Considering the expression (3.6), we obtain $\mathrm{d} \ln \rho_{a} / \mathrm{d} z=-\mathcal{D} /\left((\gamma-1) T_{a}\right)$. We can thus conclude from equation (9.3) that the shear-stress power contribution to the heat flux scales as the square of $\mathcal{D}$. This result holds near $\mathcal{D}=0$ and for the anelastic approximation. In that case, we can also apply a model of convection that Jimenez \& Zufiria (1987) obtained in the Boussinesq limit which states that $v_{z} \sim R a^{2 / 3}$. Since our parameter $R$ is proportional to $R a_{s a}$, the scaling for the shear-stress power contribution to the heat flux is found to be proportional to $R a_{s a}^{1 / 3}$, which is also the case for the global convective flux.

In Fig. 17, we plot the ratio of the compressible contribution of the convective flux to the whole convective flux (transport of enthalpy and compressible contribution). We can check that this ratio is quadratic in $\mathcal{D}$ for small values of $\mathcal{D}$. This is also nearly the case for the full compressible results, although it is not expected that the anelastic approximation should be particularly good for small values of $\mathcal{D}$. At larger values of $\mathcal{D}$, the ratio falls progressively below the quadratic trend. In terms of the Rayleigh number, although a scaling with $R a^{1 / 3}$ is hardly seen in Fig. 17, our results are consistent with a weak dependence with $R a_{s a}$.

\section{Dissipation}

The differences between the different approximations of compressible convection can also be examined in terms of the total amount of viscous dissipation compared to the convective heat flux. The Boussinesq approximation is such that the ratio of dissipation to 


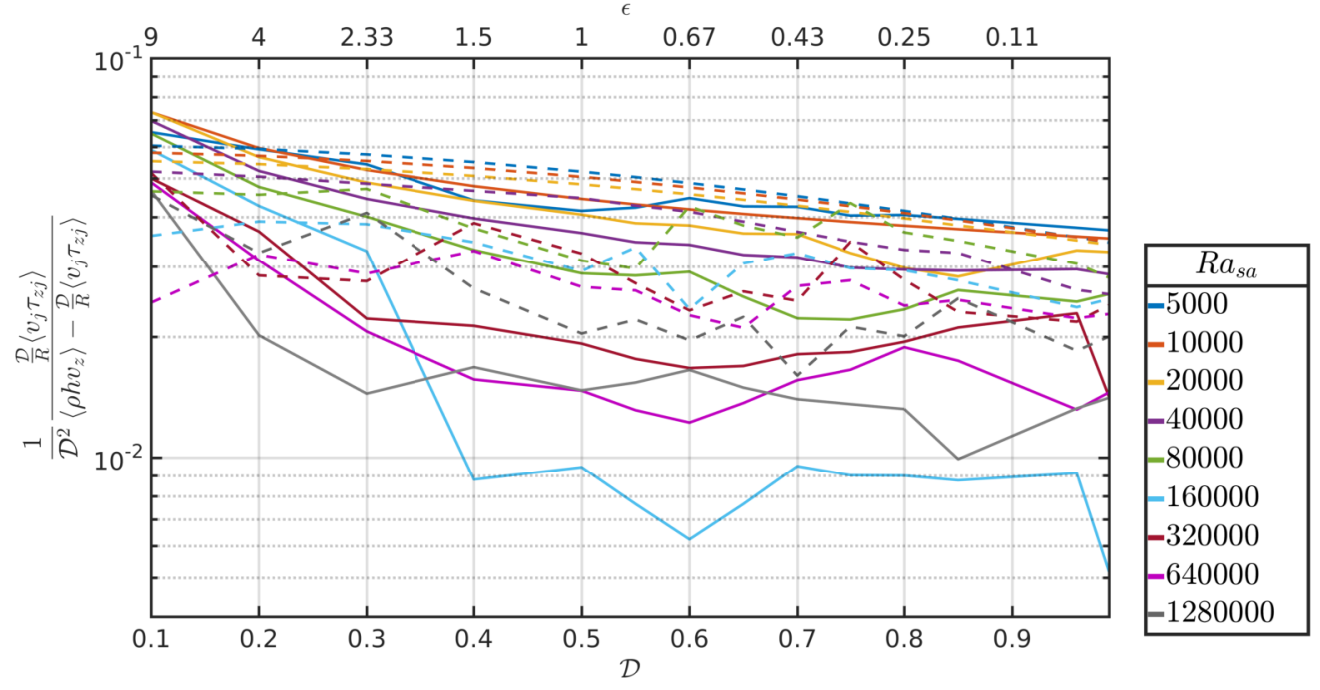

FiguRE 17. Relative weight of the shear-stress power contribution to the convected heat flux $-\mathcal{D} / R\left\langle v_{j} \tau_{z j}\right\rangle /\left(\left\langle\rho h v_{z}\right\rangle-\mathcal{D} / R\left\langle v_{j} \tau_{z j}\right\rangle\right)$ divided by $\mathcal{D}^{2}$ for the full compressible (solid lines) versus anelastic (dashed lines) calculations, for $r=3, \gamma=1.4$ and a range of values of the superadiabatic Rayleigh number $R a_{s a}$.

the convective flux can be shown to be a constant equal to the dissipation number, for all Rayleigh numbers (see Malkus (1964); Hewitt et al. (1975b) for an asymptotic derivation and for instance Doering \& Constantin (1996) for a proof within the Boussinesq model):

$$
\frac{\text { Dissipation }}{\text { Convective Flux }}=\frac{\frac{\mathcal{D}}{R}\langle\dot{\varepsilon}: \tau\rangle}{Q-\delta T}=\mathcal{D} \text {. }
$$

At larger values of $\mathcal{D}$, the ratio of viscous dissipation to the convective heat flux can be different from $\mathcal{D}$. This is now examined, depending on the nature of the model: FC, AA and ALA. We have not attempted to derive analytic expressions for this ratio for finite values of $\mathcal{D}$ because they would be derived from our results obtained on entropy sources (see sections 8 and 11.4) with an extra approximation concerning the value of temperature where dissipation occurs. Those analytical results would necessarily be more questionable than those on entropy sources.

\subsection{Dissipation: $F C$ versus $A A$}

For the anelastic approximation and for the full compressible modelling, we plot in Fig. 18 the ratio of viscous dissipation to convective heat flux divided by $\mathcal{D}$ obtained from numerical simulations. The value we obtain is close to unity corresponding to the Boussinesq limit. Globally there is a decrease of this ratio with $\mathcal{D}$ of the order of $20 \%$ for the maximal value of $\mathcal{D}=1$. Notice that near $\mathcal{D} \approx 0$ we do not get the Boussinesq ratio of 1 because even in this case we are far from a Boussinesq condition, as the temperature difference driving convection is of the same order of magnitude as the average temperature. There is no clear dependence on the superadiabatic Rayleigh number. Similarly as for the heat flux, we now plot in Fig. 19 the relative difference of the ratio of viscous dissipation to convective heat flux between the full compressible and anelastic approximation. We observe a general trend of decrease of the relative difference from slightly positive at small $\mathcal{D}$ to negative at $\mathcal{D}=1$. 


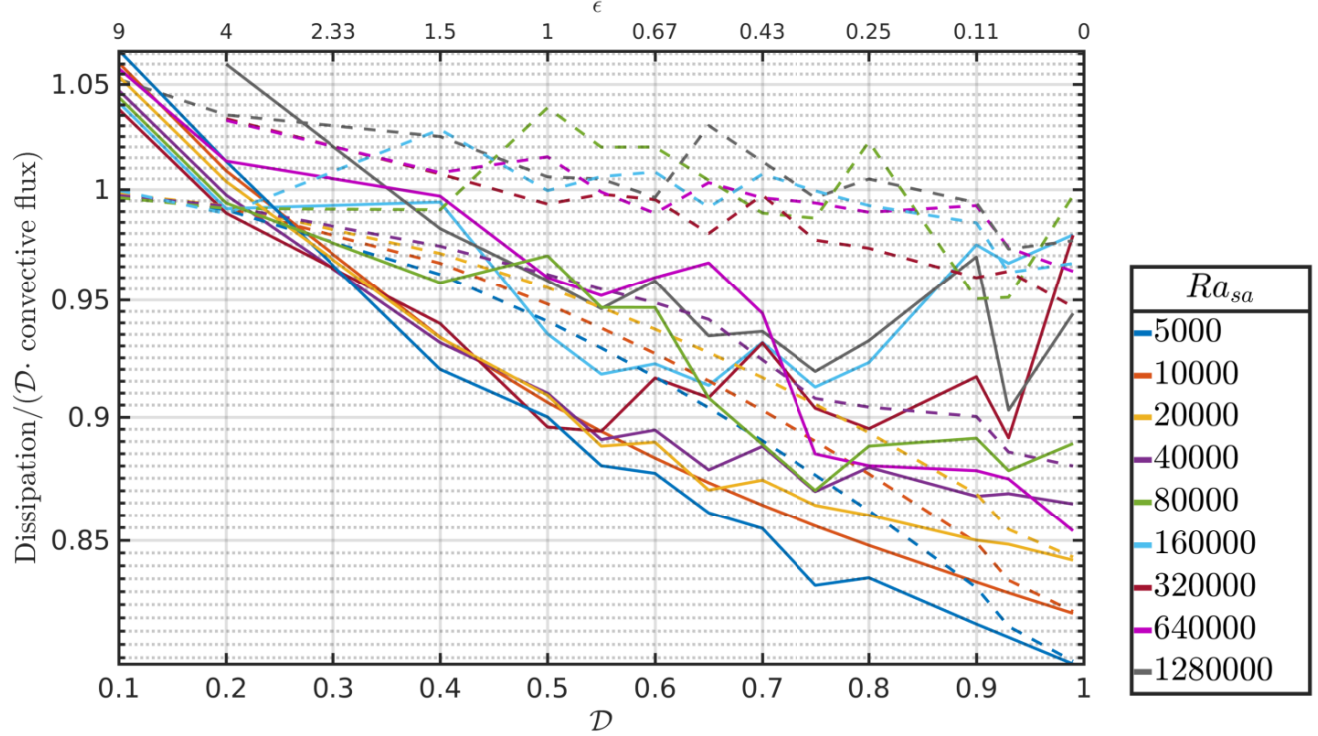

FiguRE 18. Ratio of the integral of viscous dissipation to the convective heat flux as a function of $\mathcal{D}$ for the full compressible (solid lines) versus anelastic (dashed lines) calculations, for $r=3$, $\gamma=1.4$ and a range of values of the superadiabatic Rayleigh number $R a_{s a}$.

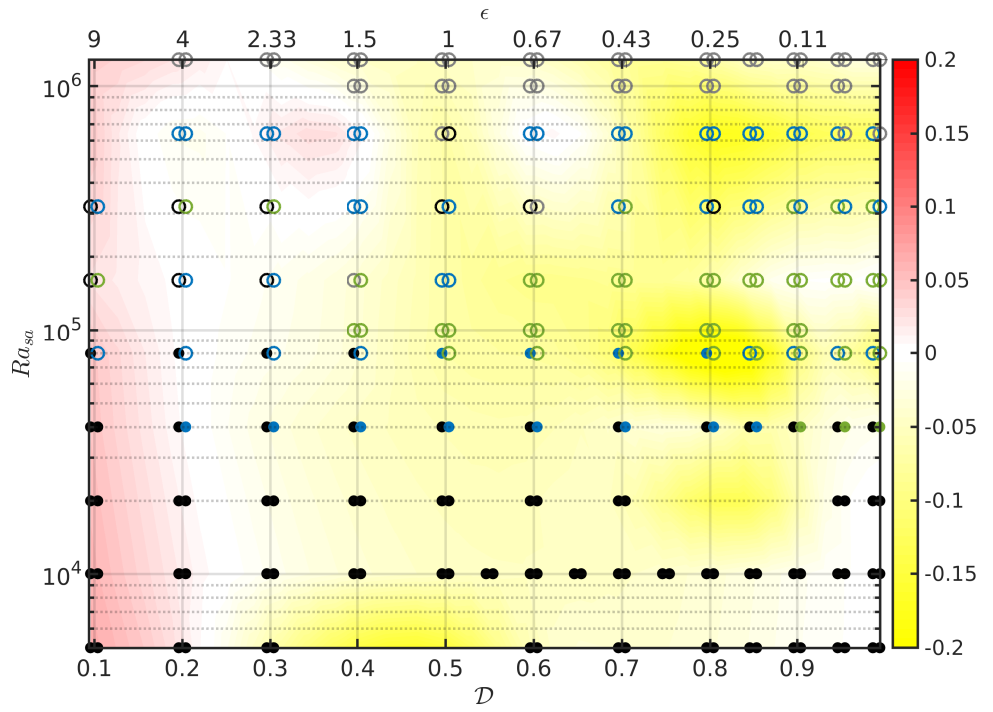

FiguRE 19. Relative difference of the ratio of the total viscous dissipation to the convective heat flux ( $\left(\right.$ ratio $^{F C}-$ ratio $\left.^{A A}\right) /$ ratio $^{F C}$ ) between the full compressible model and the anelastic approximation, in the $\mathcal{D}-R a_{s a}$ plane, for $r=3, \gamma=1.4$. The points (colored circles) follow the same code as in Fig. 14.

\subsection{Dissipation: $F C$ versus $A L A$}

The relative difference of the ratio of viscous dissipation to convective heat flux between full compressible and anelastic liquid approximation is shown in Fig. 20. The figure is rather similar to Fig. 19, so that the ratio of dissipation to heat flux is close for anelastic $\mathrm{AA}$ and anelastic liquid ALA approximations. 


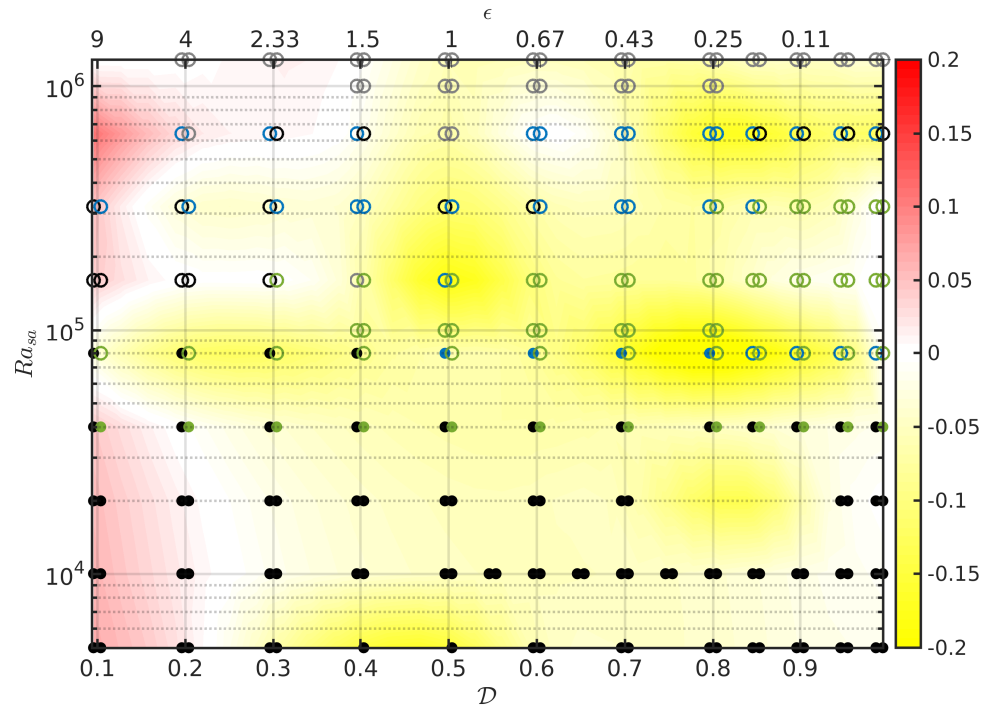

Figure 20. Relative difference of the ratio of the total viscous dissipation to the convective heat flux $\left(\left(\right.\right.$ ratio $^{F C}-$ ratio $\left.^{A L A}\right) /$ ratio $^{F C}$ ) between the full compressible and anelastic liquid approximations, in the $\mathcal{D}-R a_{\text {sa }}$ plane, for $r=3, \gamma=1.4$. The points (colored circles) follow the same code as in Fig. 14.

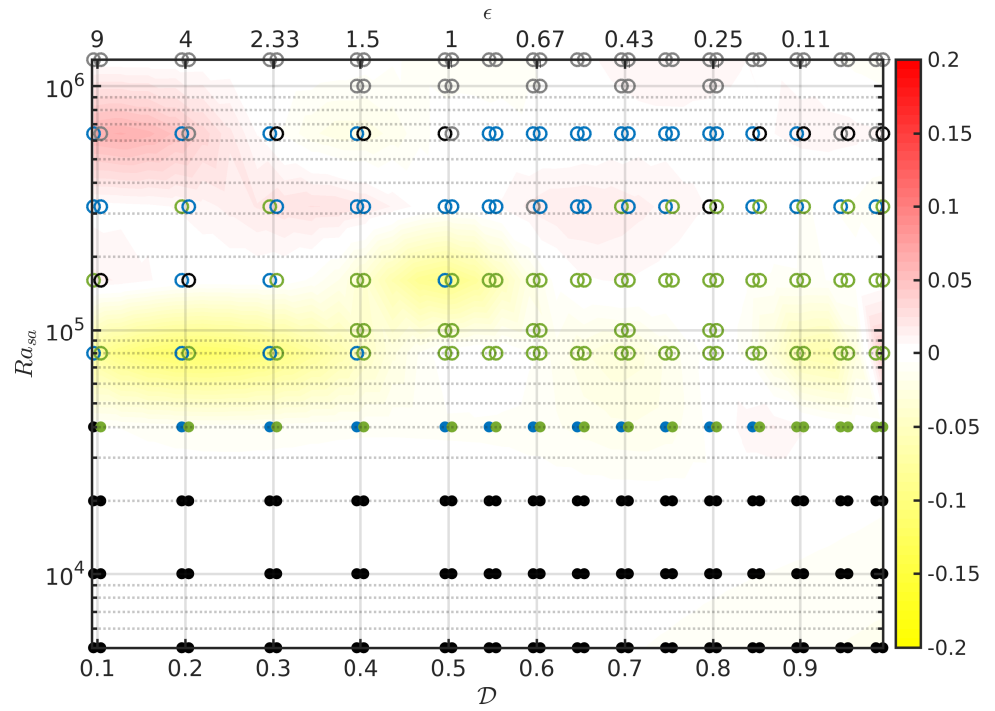

FiguRE 21. Relative difference of the ratio of the total viscous dissipation to the convective heat flux ( $\left(\right.$ ratio $^{A A}-$ ratio $\left.^{A L A}\right) /$ ratio $\left.^{A A}\right)$ between the anelastic and anelastic liquid approximations, in the $\mathcal{D}$ - $R a_{\text {sa }}$ plane, for $r=3, \gamma=1.4$. The points (colored circles) follow the same code as in Fig. 14.

\subsection{Dissipation: $A A$ versus $A L A$}

Although the computed heat fluxes are significantly larger for the anelastic approximation compared to the anelastic liquid approximation (see Fig. 16), the amount of relative viscous dissipation is surprisingly close between AA and ALA calculations, as shown in Fig. 21. 


\section{Entropy sources}

We now turn to the entropy sources, because they are building blocks of the entropy balance (see section 11.1). Evaluating the entropy sources due to conduction can eventually provide an estimate for the entropy sources due to viscous dissipation. This is how dissipation (viscous or ohmic) has been inferred for the Earth's core (Braginsky \& Roberts 1995). In this section, we evaluate the difference of entropy sources obtained with anelastic and full compressible models, and derive bounds for the entropy sources (in section 11.4) in the anelastic approximation.

\subsection{Entropy balance}

From the point of view of the entropy equation (2.11), it may seem that the anelastic approximation (see Table 1 ) is justified as long as $T$ and $\rho$ become close to $T_{a}$ and $\rho_{a}$. This condition on $T$ is easily expressed using the parameter $\epsilon$ defined in equation (2.19), as $\epsilon \ll 1$. It has been shown, for viscous flows (Bercovici et al. 1992; Ricard 2015), that the corresponding condition on $\rho$ can be expressed as $M^{2} \operatorname{Pr} R a_{s a}^{-1} \ll 1$. This last expression is indeterminate with an infinite Prandtl number and zero Mach number. However, using a viscous velocity estimate from Stokes equation, $v \sim\left(\rho_{0} g \alpha_{0} \Delta T_{s a} L^{2}\right) / \eta$, using the expression of the sound velocity $\sqrt{K_{s} / \rho_{0}}$ and Mayer's equation $c_{p}-c_{v}=$ $\left(\alpha^{2} K_{s} T\right) /(\rho \gamma)$, that condition for small relative density fluctuations can be expressed as

$$
\epsilon \hat{\alpha}^{2} \mathcal{D}^{2} /(\gamma-1) \ll 1,
$$

which can be evaluated from our set of dimensionless numbers, but which is independent of the superadiabatic Rayleigh number.

However, the perspective is changed when the global entropy balance is considered. From (2.11), dividing by $T$ and integrating over the volume and time leads to the following entropy balance in statistically stationary cases: the left-hand side integrates to zero and the last term on the right-hand side is integrated by parts, providing the 'reversible' entropy exchange with heat sources and the 'irreversible' entropy source due to thermal conduction.

$$
Q\left(\frac{1}{T_{t o p}}-\frac{1}{T_{b o t}}\right)=\frac{\mathcal{D}}{R}\left\langle\frac{\dot{\varepsilon}: \tau}{T}\right\rangle+\left\langle\frac{\nabla T \cdot \nabla T}{T^{2}}\right\rangle,
$$

where $Q$ denotes the average dimensionless heat flux across the layer, $Q=-\bar{\partial}_{z} T_{z=0}=$ $-\bar{\partial}_{z} T_{z=1}$, which is exactly the same heat flux $Q$ as defined in equation (9.1). Now, when the anelastic entropy equation (see Table 1 ) is divided by $T_{a}$ and integrated over volume and time, a different equation emerges:

$$
Q\left(\frac{1}{T_{a}(d)}-\frac{1}{T_{a}(0)}\right)=\frac{\mathcal{D}}{R}\left\langle\frac{\dot{\varepsilon}: \tau}{T_{a}}\right\rangle+\left\langle\frac{\nabla T_{a} \cdot \nabla T}{T_{a}^{2}}\right\rangle,
$$

Those two expressions of entropy balance are different. One aspect of that difference is related to the denominator temperatures, but obviously this aspect becomes negligible as $\epsilon$ becomes very small compared to one, $\epsilon \ll 1$. Even when this is the case, the expressions for the source of entropy due to thermal conduction are different: the exact expression (11.2) contains the integral of the square of superadiabatic temperature gradients, while the anelastic expression (11.3) does not.

\subsection{Viscous entropy sources}

We plot in Fig. 22 (respectively 23) the ratio of the integral of viscous entropy sources $\mathcal{D}\langle\epsilon: \tau / T\rangle / R$ to $\mathcal{D}$ times the heat flux using the temperature $T$ (respectively $T_{a}$ ), $i$. $e$. 


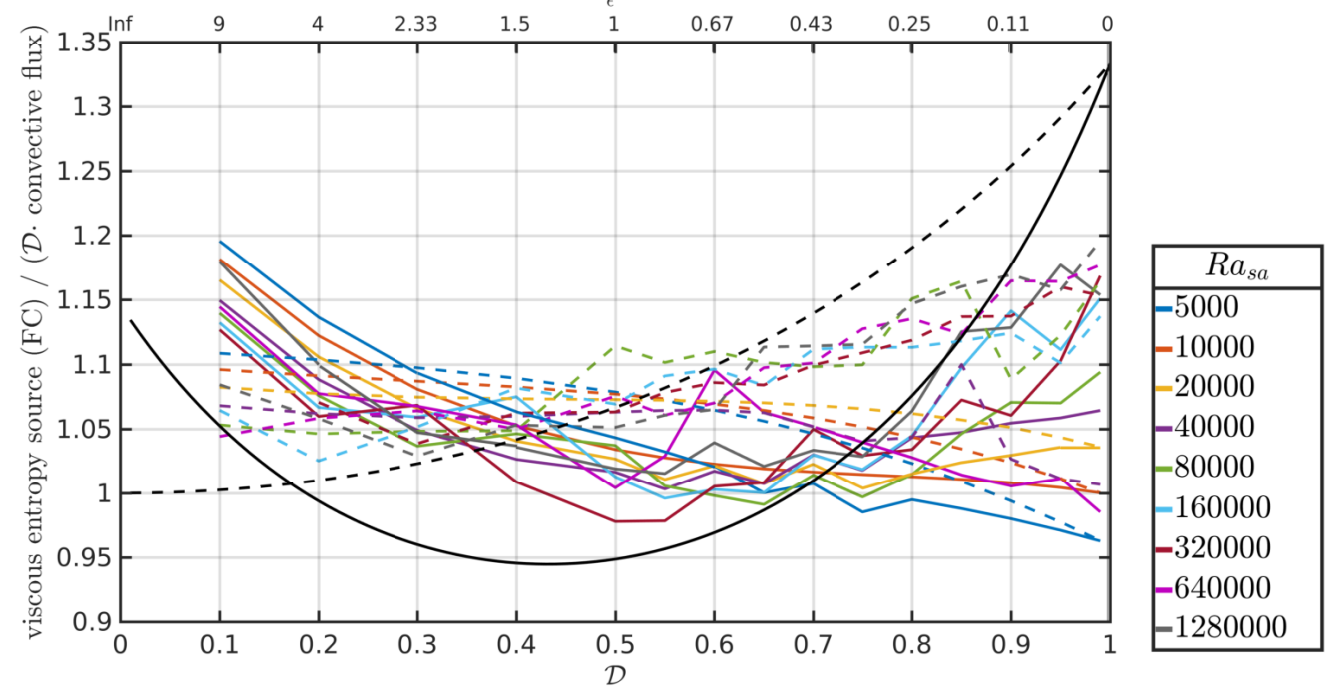

FiguRE 22. Ratio of total viscous entropy sources, $\mathcal{D} / R\langle(\dot{\epsilon}: \tau) / T\rangle$ (using temperature $T$ ) to the convective heat flux, divided by $\mathcal{D}$ as a function of $\mathcal{D}$ for the full compressible (solid lines) versus anelastic (dashed lines) calculations, for $r=3, \gamma=1.4$ and a range of values of the superadiabatic Rayleigh number $R a_{s a}$. Black curves correspond to the anelastic (dashed) and full compressible (solid) analytical expressions (11.7) and (11.8).

$\langle\epsilon: \tau / T\rangle /\left(R Q_{s a}\right)$ (respectively $\left\langle\epsilon: \tau / T_{a}\right\rangle /\left(R Q_{s a}\right)$ ). For small values of $\mathcal{D}$ it is expected that this last ratio will converge to 1 , as the viscous entropy source becomes identical to viscous dissipation (as $T_{a}$ converges everywhere to 1 ). This can indeed be seen in Fig. 23. The viscous entropy sources of the full compressible model are slightly larger than those of the anelastic model at small $\mathcal{D}$ (for the same convective flux). This changes above $\mathcal{D}=0.2$ (or 0.3 ) in Fig. 23 (or 22) where the full compressible viscous entropy sources are smaller than their anelastic counterparts. Near the maximum value of $\mathcal{D}$, we observe a convergence of the full compressible and anelastic curves, but the ratio is not equal to one and depends on the superadiabatic Rayleigh number. This convergence is somehow expected, as the anelastic approximation is at its best when the adiabatic gradient is responsible for the largest possible part of the temperature difference between the top and bottom. Equivalently, this means that the superadiabatic temperature departures are very small compared to the temperatures of the adiabatic profile.

Using the simplified temperature profile (7.1), the entropy balances (anelastic and full compressible) and our rationale, we can also retrieve some features of the numerical solutions regarding the viscous source of entropy. From this profile, we can determine the conduction entropy sources $(\boldsymbol{\nabla} T \cdot \boldsymbol{\nabla} T) / T^{2}$ and $\left(\boldsymbol{\nabla} T \cdot \boldsymbol{\nabla} T_{a}\right) / T_{a}^{2}$ (FC and AA). In fact at high superadiabatic Rayleigh number, one has $\delta_{t} \ll 1, \delta_{b} \ll 1, Q \gg \mathcal{D}$ and therefore only the gradients in the boundary layers of equation (7.1) contribute to these terms,

$$
\begin{aligned}
\left\langle\frac{\nabla T \cdot \nabla T}{T^{2}}\right\rangle & \approx \frac{4 Q \delta T}{4-\delta T^{2}}+\frac{Q}{1+\delta T / 2-\delta_{b} Q}-\frac{Q}{1-\delta T / 2+\delta_{t} Q}, \\
\left\langle\frac{\nabla T \cdot \nabla T_{a}}{T_{a}^{2}}\right\rangle & \approx \frac{4 Q \mathcal{D}}{4-\mathcal{D}^{2}}+\frac{Q}{1+\mathcal{D}\left(\frac{1}{2}-\delta_{b}\right)}-\frac{Q}{1-\mathcal{D}\left(\frac{1}{2}-\delta_{t}\right)} .
\end{aligned}
$$

From these expressions and from the entropy balances (FC and AA in equations (11.2) 


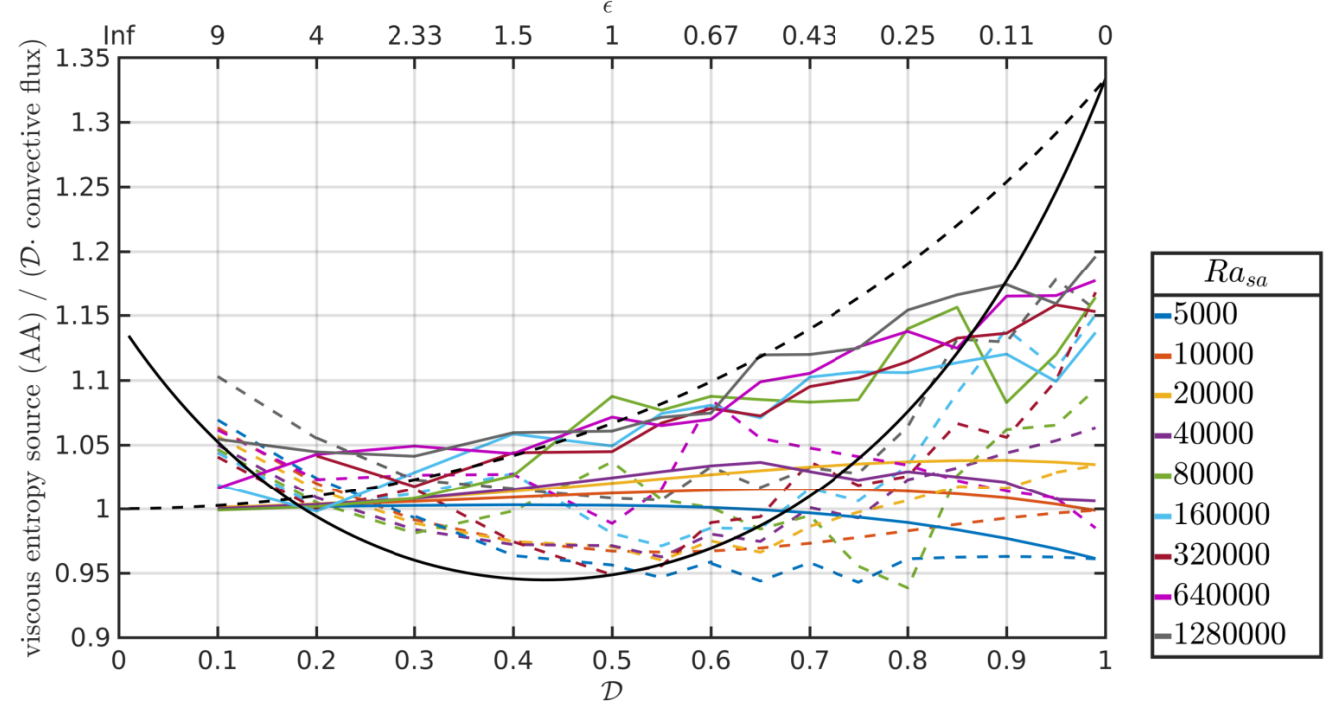

FigURE 23. Ratio of the integral of viscous entropy sources, $\mathcal{D} / R\left\langle(\dot{\epsilon}: \tau) / T_{a}\right\rangle$ (using the adiabatic temperature profile $T_{a}$ ), to the convective heat flux divided by $\mathcal{D}$ as a function of $\mathcal{D}$ for the full compressible (solid lines) versus anelastic (dashed lines) calculations, for $r=3, \gamma=1.4$ and a range of values of the superadiabatic Rayleigh number $R a_{s a}$.

and (11.3) respectively), we obtain the viscous dissipation entropy sources (FC and AA).

$$
\begin{aligned}
& \frac{\mathcal{D}}{R}\left\langle\frac{\dot{\epsilon}: \tau}{T}\right\rangle /\left.Q_{\text {conv }}\right|_{F C} \simeq-\frac{1}{1+\delta T / 2-\delta_{b} Q}+\frac{1}{1-\delta T / 2+\delta_{t} Q}, \\
& \frac{\mathcal{D}}{R}\left\langle\frac{\dot{\epsilon}: \tau}{T_{a}}\right\rangle /\left.Q_{\text {conv }}\right|_{A A} \simeq-\frac{1}{1+\mathcal{D} / 2}+\frac{1}{1-\mathcal{D} / 2},
\end{aligned}
$$

where the limit of large Nusselt number $\left(\delta_{t}<<1, \delta_{b}<<1\right.$ and $\left.Q_{\text {conv }}=Q-\delta T \approx Q\right)$ has been taken. As $Q=\delta T_{\text {sab }} / \delta_{b}=\delta T_{\text {sat }} / \delta_{t}$, using (8.1) and (8.13), with $\delta T=2(r-1) /(r+1)$ and $\delta T_{s a}=\delta T-\mathcal{D}$, we can also express the viscous source of entropy dissipation for the full compressible case, as

$$
\frac{\mathcal{D}}{R}\left\langle\frac{\dot{\epsilon}: \tau}{T}\right\rangle /\left.Q_{\text {conv }}\right|_{F C} \simeq-\frac{1}{1+\frac{r-1}{r+1} \frac{x-1}{x+1}+\frac{\mathcal{D}}{1+x}}+\frac{1}{1+\frac{r-1}{r+1} \frac{x-1}{x+1}-\frac{\mathcal{D} x}{1+x}} .
$$

These expressions (solid and dashed lines in Figs. 22 and 23) basically mimic the behaviour of the numerical results. In particular the fact that the $\mathrm{FC}$ curve does not converge towards unity as $\mathcal{D}$ converges towards zero is due to the asymmetry of the temperature profile $x \neq 1$ (see expression (11.8)).

\subsection{Entropy balance and convergence criterion for anelastic models}

Now, all terms in equation (11.2) and (11.3) can be evaluated for a numerical calculation, should it be full compressible or obtained using an anelastic model. It is expected that (11.2) will be satisfied by full compressible calculations and (11.3) by anelastic calculations. The other equation is, in general, not satisfied and will now be used to build an intrinsic measure of the distance between the full compressible and anelastic 
models. Let us defined the following functionals:

$$
\begin{aligned}
d_{F C}= & \frac{Q\left(\frac{1}{T_{t o p}}-\frac{1}{T_{b o t}}\right)-\frac{\mathcal{D}}{R}\left\langle\frac{\dot{\varepsilon}: \tau}{T}\right\rangle-\left\langle\frac{\nabla T \cdot \nabla T}{T^{2}}\right\rangle}{Q\left(\frac{1}{T_{t o p}}-\frac{1}{T_{b o t}}\right)}, \\
d_{A A}= & \frac{Q\left(\frac{1}{T_{a}(d)}-\frac{1}{T_{a}(0)}\right)-\frac{\mathcal{D}}{R}\left\langle\frac{\dot{\varepsilon}: \tau}{T_{a}}\right\rangle-\left\langle\frac{\nabla T_{a} \cdot \nabla T}{T_{a}^{2}}\right\rangle}{Q\left(\frac{1}{T_{a}(d)}-\frac{1}{T_{a}(0)}\right)} .
\end{aligned}
$$

Suppose that some numerical calculation AA is done with the anelastic model: we expect $d_{A A}(A A)=0$ up to the numerical errors. Moreover $d_{F C}(A A)$ provides an intrinsic measurement of the discrepancy of the numerical solution to an ideal full compressible solution. A necessary condition for the anelastic results to be considered a good representation of full compressible convection is that they nearly satisfy the full compressible entropy balance, i.e. that $d_{F C}(A A)$ is small. This is an intrinsic measure of the quality of the anelastic approximation, because no numerical solution of the full compressible equations is needed. The converse is true actually: for a given numerical solution $\mathrm{FC}$ of the full compressible equations, we have $d_{F C}(F C)=0$ (up to numerical errors), and $d_{A A}(F C)$ provides an intrinsic measure of how well the anelastic entropy balance is satisfied, i.e. how far it lies from an anelastic solution.

On figure 24, we plot the expressions (11.9) and (11.10) for a set of numerical solutions of the full compressible FC and anelastic AA models. In fact, the absolute value of these quantities are plotted, but we have observed that $d_{A A}(F C)$ is positive and $d_{F C}(A A)$ is negative: this was expected since the full compressible source of entropy due to thermal conduction is larger than its anelastic counterpart, as it takes into account the square of the superadiabatic temperature gradients. It is particularly interesting to consider the small values of $\epsilon$ : in this limit, we observe that $d_{F C}(A A)$ and $d_{A A}(F C)$ collapse on a single curve when they are plotted as a function of $\epsilon^{2} R a_{s a}^{1 / 3}$ which is a proxy for $\epsilon q$, where $q=Q_{s a} / Q_{a}$. For a given superadiabatic heat flux ratio $q$, making the superadiabatic temperature ratio $\epsilon$ small reduces the distance between the full-compressible FC and anelastic AA entropy sources.

As can be seen in Fig. 24, those distances obey approximately the following relationship

$$
d_{F C}(A A) \simeq d_{A A}(F C) \simeq 2 \times 10^{-2} \epsilon^{2} R a_{s a}^{1 / 3} \simeq 2 \times 10^{-2} \epsilon q
$$

while the anelastic liquid approximation has a different scaling

$$
d_{F C}(A L A) \simeq 4 \times 10^{-4} \epsilon R a_{s a}^{1 / 6} \simeq 4 \times 10^{-4}(\epsilon q)^{1 / 2} .
$$

Although the convergence towards zero is asymptotically faster for the anelastic results AA, it turns out that, within the range of parameters actually computed, the distance $d_{F C}(A L A)$ is smaller than $d_{F C}(A A) \simeq d_{A A}(F C)$.

Concerning the anelastic approximation AA, the convergence of entropy sources seen in Fig. 24 can be associated to a convergence of the heat fluxes with full compressible results. We had indeed observed in Fig. 14 that as the dissipation number approaches its maximum value (implying a small value for $\epsilon$ ) the relative difference of heat fluxes between $\mathrm{AA}$ and FC is small, particularly at low values of $R a_{s a}$ (hence small $q$ ). On the contrary, such a convergence is not seen in Fig. 15 between ALA and FC: the relative difference of heat fluxes increases when $\mathcal{D}$ increases, even though entropy sources 


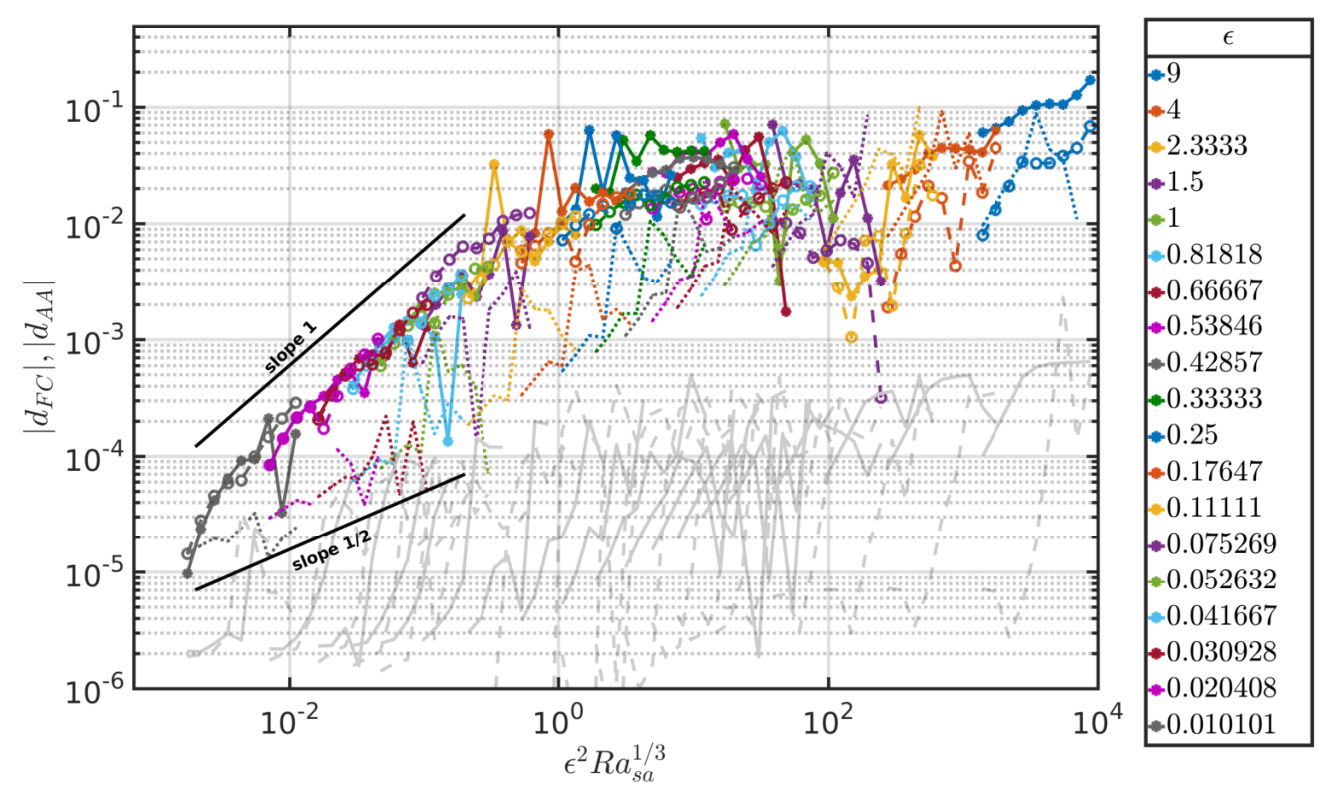

Figure 24. Quantities (11.9) and (11.10) are plotted against $\epsilon^{2} R a_{s a}^{1 / 3}$ for three types of calculations: full compressible FC, anelastic AA and anelastic liquid ALA. The empty circles, $d_{F C}(A A)$, connected with dashed lines of various colors, indicate the distance of the anelastic calculations to the full compressible entropy balance, while the full circles, $d_{A A}(F C)$, connected with colored solid lines, correspond to the distance of the full compressible calculations to the anelastic entropy balance. The colored dotted lines correspond to the distance of the anelastic liquid calculations to the full compressible entropy balance, i.e. $d_{F C}(A L A)$. The pale gray lines represent the level of numerical errors associated with the full compressible calculations $d_{F C}(F C)$ (solid) and anelastic calculations $d_{A A}(A A)$ (dashed) regarding the corresponding entropy balance.

converge (distance $d_{F C}(A L A)$ is small).

\subsection{Bounds on anelastic entropy sources}

In the anelastic approximations, AA and ALA, according to (11.3), the source of entropy associated with irreversible thermal conduction is

$$
\left\langle\frac{\nabla T_{a} \cdot \nabla T}{T_{a}^{2}}\right\rangle .
$$

This does not constitute a very satisfactory entropy source as it is not guaranteed to be positive: whether it might be physically relevant or not, one can easily imagine a temperature field making the integrand of (11.13) locally negative (e.g. in the regions close to the boundary layers where the temperature profile overshoots the adiabatic gradient). This was already pointed out in Braginsky \& Roberts (1995), page 35. However, we will now derive upper and lower bounds for that expression (11.13), which will then allow us to obtain lower and upper bounds for the source of entropy associated with irreversible viscous dissipation. In our derivation, we make an assumption concerning the temperature field: it is assumed to stay close to the adiabat, precisely within $\pm 1 / 2 \Delta T_{s a}$ which for all the experiments and simulations that we are aware of, seems to be a very conservative assumption. 
Using the decomposition $T=T_{a}+T^{\prime}$, we have

$$
\left\langle\frac{\nabla T \cdot \nabla T_{a}}{T_{a}^{2}}\right\rangle=\left\langle\frac{\left[\frac{\mathrm{d} T_{a}}{\mathrm{~d} z}\right]^{2}}{T_{a}^{2}}\right\rangle+\left\langle\frac{\frac{\partial T^{\prime}}{\partial z} \frac{\mathrm{d} T_{a}}{\mathrm{~d} z}}{T_{a}^{2}}\right\rangle .
$$

Integrating the second term by parts leads to

$$
\left\langle\frac{\nabla T \cdot \nabla T_{a}}{T_{a}^{2}}\right\rangle=\left\langle\frac{\left[\frac{\mathrm{d} T_{a}}{\mathrm{~d} z}\right]^{2}}{T_{a}^{2}}\right\rangle+\frac{\Delta T_{s a}}{2}\left(\frac{Q_{a b}}{T_{a b}^{2}}+\frac{Q_{a t}}{T_{a t}^{2}}\right)+\left\langle T^{\prime} \frac{\mathrm{d}^{2}}{\mathrm{~d} z^{2}}\left(\frac{1}{T_{a}}\right)\right\rangle,
$$

where $T_{a b}=T_{a}(z=0)$ and $T_{a t}=T_{a}(z=1)$ are the bottom and top dimensionless adiabatic temperatures and $Q_{a b}=-\left(\mathrm{dT}_{\mathrm{a}} / \mathrm{d} z\right)_{z=0}$ and $Q_{a t}=-\left(\mathrm{dT}_{\mathrm{a}} / \mathrm{d} z\right)_{z=1}$ are the dimensionless heat fluxes down the adiabat at the bottom and top of the layer. Using Schwartz inequality and our assumption on $T^{\prime}$, the last integral can be bounded so that the conduction source of entropy is bounded above and below by

$$
\left\langle\frac{\nabla T \cdot \nabla T_{a}}{T_{a}^{2}}\right\rangle=\int_{0}^{1} \frac{\left[\frac{\mathrm{d} T_{a}}{\mathrm{~d} z}\right]^{2}}{T_{a}^{2}} d z+\frac{\Delta T_{s a}}{2}\left(\frac{Q_{a b}}{T_{a b}^{2}}+\frac{Q_{a t}}{T_{a t}^{2}}\right) \pm \frac{\Delta T_{s a}}{2} \sqrt{\int_{0}^{1}\left[\frac{\mathrm{d}^{2}}{\mathrm{~d} z^{2}}\left(\frac{1}{T_{a}}\right)\right]^{2} d z}
$$

Considering the anelastic entropy balance (11.3), the dissipation source of entropy is then bounded above and below in the following way

$$
\frac{\mathcal{D}}{R}\left\langle\frac{\dot{\epsilon}: \tau}{T_{a}}\right\rangle=\frac{Q}{T_{a t}}-\frac{Q}{T_{a b}}-\int_{0}^{1} \frac{\left[\frac{\mathrm{d} T_{a}}{\mathrm{~d} z}\right]^{2}}{T_{a}^{2}} d z-\frac{\Delta T_{s a}}{2}\left(\frac{Q_{a b}}{T_{a b}^{2}}+\frac{Q_{a t}}{T_{a t}^{2}}\right) \pm \frac{\Delta T_{s a}}{2} \sqrt{\int_{0}^{1}\left[\frac{\mathrm{d}^{2}}{\mathrm{~d} z^{2}}\left(\frac{1}{T_{a}}\right)\right]^{2} d z}
$$

Now, in the particular case of the ideal gas equation of state, we have $T_{a}=1-\mathcal{D}\left(z-\frac{1}{2}\right)$ and the bounds above can be written as follows

$$
\begin{aligned}
\left\langle\frac{\nabla T \cdot \nabla T_{a}}{T_{a}^{2}}\right\rangle & =Q_{a} \frac{\mathcal{D}}{1-\frac{\mathcal{D}^{2}}{4}}+\frac{\mathcal{D} Q_{s a}}{N u_{s a}} \frac{1+\frac{\mathcal{D}^{2}}{4}}{\left(1-\frac{\mathcal{D}^{2}}{4}\right)^{2}} \pm \frac{\mathcal{D}^{2} Q_{s a}}{N u_{s a}} \sqrt{\frac{1+\frac{\mathcal{D}^{2}}{2}+\frac{\mathcal{D}^{4}}{80}}{\left(1-\frac{\mathcal{D}^{2}}{4}\right)^{5}}} \\
\frac{\mathcal{D}}{R}\left\langle\frac{\dot{\epsilon}: \tau}{T_{a}}\right\rangle & =Q_{s a}\left[\frac{\mathcal{D}}{1-\frac{\mathcal{D}^{2}}{4}}-\frac{\mathcal{D}}{N u_{s a}} \frac{1+\frac{\mathcal{D}^{2}}{4}}{\left(1-\frac{\mathcal{D}^{2}}{4}\right)^{2}} \mp \frac{\mathcal{D}^{2}}{N u_{s a}} \sqrt{\frac{1+\frac{\mathcal{D}^{2}}{2}+\frac{\mathcal{D}^{4}}{80}}{\left(1-\frac{\mathcal{D}^{2}}{4}\right)^{5}}}\right]
\end{aligned}
$$

where the superadiabatic Nusselt number, defined as $N u_{s a}=Q_{s a} / \Delta T_{s a}$, was already introduced in (8.11). It can be observed here that a Boussinesq result on dissipation is recovered from the anelastic dissipation in the limit of vanishing $\mathcal{D}$. From Boussinesq equations, viscous dissipation is exactly equal to the dissipation number times the convective heat flux. Here, the bracket on the right-hand side of (11.19) converges towards $\mathcal{D}-\mathcal{D} / N u_{s a}$ plus higher order terms. Now the factor $Q_{s a}=Q-\mathcal{D}$ is expanded at small $\mathcal{D}$ as $Q-\delta T+\delta T-\mathcal{D} \simeq Q_{\text {conv }}+\delta T=Q_{\text {conv }}(1+1 / N u)$. Hence equation (11.19) converges towards the Boussinesq limit (10.1).

We finally compare the approximate expression (11.7) with the exact bound (11.19). They are quite similar: in the large bracket of (11.19) we find the same expression as in the right-hand side of (11.7), plus a term proportional to $\mathcal{D} / N u_{s a}$, with an uncertainty within a term proportional to $\pm \mathcal{D}^{2} / N u_{s a}$. However, the main difference is due to the reference heat flux: it is $Q_{c o n v}$ for (11.7) and $Q_{s a}$ for (11.19). As $Q_{c o n v}=Q-\delta T$ and $Q_{s a}=Q-\mathcal{D}$, we have $Q_{c o n v}=Q_{s a}-\delta T_{s a}=Q_{s a}\left(1-1 / N u_{s a}\right)$. This means that the approximate expression (11.7) has a relative error of order $N u_{s a}^{-1}$ compared to the exact bound (11.19), which is to be expected: first, we have assumed that the temperature 
field is given by our simplified model (7.1) with $\beta=0$ for this anelastic case, secondly we have neglected the temperature gradients in the core of the flow compared to those in the boundary layers when deriving (11.7).

\section{Conclusions}

We have been investigating numerically some features of compressible convection and the differences induced when an anelastic or an anelastic liquid approximation is used. In order to make the problem more tractable, we have decided to remove some difficulties. First, we have considered the case of an infinite Prandtl number. We have shown that this makes the Mach number zero and eliminates sound waves. So, any difference observed when using approximate models cannot be attributed to sound waves, but are due to compressibility (dissipation number) and finite ratio of imposed temperatures. Secondly, we have restricted our analysis to ideal gases. The advantage of this type of equations of state is that the adiabatic temperature gradient is uniform (under a uniform gravity field) so that we do not have to treat cases of stable subadiabatic regions appearing when conduction alone is able to carry the whole heat flux while the rest of the fluid domain is convecting: the depth of the convective zone is always equal to the height of the fluid domain. As a consequence, we clearly define an academic problem as probably no fluid will follow an ideal gas equation of state and have a very large Prandtl number. Yet, this defines a sound physical problem and we believe that it is of interest to study the effect of compressibility on convection using the exact (full compressible) equations or anelastic approximations.

As the conditions depart from the Boussinesq limit (temperature ratio significantly larger than unity, non negligible dissipation number), we observe that an asymmetry develops between top and bottom thermal boundary layers. This effect is not specific to compressible convection and is also observed when the dissipation number is very small (Wu \& Libchaber 1991): in that case, one might call it a non-Oberbeck-Boussinesq effect. However, we notice that this asymmetry is enhanced (and in the opposite direction) when the dissipation number is increased. We have suggested a rationale, based on a constant time of boundary layer development (for a given superadiabatic Rayleigh number), which

ultimately relates the thickness of a boundary layer to the inverse of the square-root of density (because we have a uniform thermal conductivity, thermal diffusivity is inversely proportional to density). Temperature affects density, but pressure has an even larger impact on density for a dissipation number of order unity, particularly when $\gamma-1$ is small. We have shown that a consequence of this asymmetry is that it has an impact on the entropy sources: the entropy source due to thermal conduction is affected because a temperature gradient in a small volume element has a larger contribution at a lower temperature (within the top boundary layer) than at higher temperature (within the bottom boundary layer). Any impact on the entropy source due to conduction is then transferred to the entropy source due to viscous dissipation. Using our rationale on boundary layer development, we have derived a prediction for the integral of entropy sources, different for the full compressible and anelastic cases, in a good agreement with our numerical results.

Concerning the heat flux, we have shown that the anelastic models underestimate it systematically by 10 to $30 \%$ (up to $40 \%$ for the anelastic liquid approximation). This is partly due to the transition between steady to unsteady convection, taking place systematically at lower superadiabatic Rayleigh numbers for the anelastic approximations. Here again, our rationale was used to derive predictions for the heat flux, showing a lower heat flux in the anelastic approximation, in good agreement with the numerical results (see 
Fig. 11). In a symmetric top/bottom expression of the Nusselt number (8.16), we show that the product of top and bottom densities $\rho_{t} \rho_{b}$ plays a role: this can also be found with a different scaling in Tilgner (2011), although inertia, rather than compressibility, is important in this work and a rationale based on equal top and bottom kinetic energy densities from free-fall velocity is proposed.

In the heat flux, we have studied particularly the typical compressible contribution due to the power of shear stress. We have shown that it scales quadratically in the dissipation number $\mathcal{D}$ and that its relative magnitude compared to the classical convective flux depends weakly on the superadiabatic Rayleigh number.

Entropy sources have been considered with attention. In the anelastic case, we have derived upper and lower bounds for the conduction and viscous entropy sources, which are accurate when the Nusselt number is large. We have not been able to do so for the full compressible case. Instead, we have introduced a tool to evaluate intrinsically a distance between full compressible and anelastic approximations. That tool is based on the entropy balance. The full compressible entropy balance is different from the anelastic entropy balance, but all terms in these balances can be evaluated after each numerical simulation (full compressible or anelastic). One of these balances is satisfied (up to numerical errors) and the other one tells us how far full compressible results are from anelastic results. We obtain a good collapse on a single curve when that distance is plotted against the product of the superadiabatic temperature difference ratio and the superadiabatic heat flux ratio $\epsilon q$ (or $\epsilon^{2} R a_{s a}^{1 / 3}$ ). Our numerical solutions in the anelastic liquid approximation are closer to satisfying the full compressible entropy balance than the anelastic results, however the convergence seems to be slower.

On many outcomes of the numerical simulations, we have observed that the anelastic $\mathrm{AA}$ and full compressible FC results agree quite well when the superadiabatic temperature difference is very small compared to the adiabatic temperature difference (i.e. when the dissipation number approaches the maximal value authorized by the imposed temperature ratio). This is consistent with the convergence mentioned above regarding entropy balances, although the convergence is made more difficult to observe at large superadiabatic Rayleigh numbers. This is however not the case for the anelastic liquid approximation ALA, which we expect to be less good when $\mathcal{D}$ increases (see Anufriev et al. (2005)).

Here, we have restricted our study to ideal gases, but we plan to investigate other equations of state, in particular with a small $\alpha T$ product, which are better suited to condensed matter. The anelastic liquid approximation is supposed to be better in such a case.

It has become apparent during this study that thermal diffusivity is crucial to determine the relative thickness of each boundary layer. It will be interesting to perform numerical simulations with a fluid of uniform thermal diffusivity, by adjusting thermal conductivity to density and specific heat capacity $c_{p}$. In that case, however, the temperature drop will be different across the top and bottom boundary layers, since thermal conductivity will no longer be uniform while the conservation of energy will continue to impose an equal top and bottom conduction heat flux.

\section{Acknowledgements}

We thank P2CHPD for computing facilities. The authors are grateful to the LABEX Lyon Institute of Origins (ANR-10-LABX-0066) of the Université de Lyon for its financial support within the program "Investissements d'Avenir" (ANR-11-IDEX-0007) of the French government operated by the National Research Agency (ANR). JC also ac- 
knowledges support from MINECO grant MTM2014-56392-R and ICMAT-Severo Ochoa project SEV-2015-0554.

\section{REFERENCES}

Alboussière, Thierry \& Ricard, Yanick 2013 Reflections on dissipation associated with thermal convection. Journal of Fluid Mechanics 725, 1469-7645.

AlboussiÈre, Thierry \& Ricard, YAnick 2017 Rayleigh-Bénard stability and the validity of quasi-Boussinesq or quasi-anelastic liquid approximations. Journal of Fluid Mechanics 817, 264-305.

Anufriev, A.P., Jones, C.A. \& Soward, A.M. 2005 The Boussinesq and anelastic liquid approximations for convection in the Earth's core. Phys. Earth and Planet. Int. 152, 163-190.

Bercovici, D., Schubert, G. \& Glatzmaier, G.A. 1992 Three-dimensional convection of an infinite-Prandtl-number compressible fluid in a basally heated spherical shell. J. Fluid Mech. 239, 683-719.

Boussinesq, J. 1903 Théorie analytique de la chaleur, tome 2, pp. 157-161. Paris: GauthierVillars.

Braginsky, S.I. \& Roberts, P.H. 1995 Equations governing convection in earth's core and the geodynamo. Geophys. Astrophys. Fluid Dynam. 79, 1-97.

Davis, Tiмотну A. 2004 Algorithm 832: Umfpack v4.3-an unsymmetric-pattern multifrontal method. Trans. Math. Softw. 30 (2), 196-199.

Davis, Timothy A. 2006 Direct Methods for Sparse Linear Systems. Philadelphia, PA, USA: SIAM.

Doering, C. R. \& Constantin, P. 1996 Variational bounds on energy dissipation in incompressible flows. III. Convection. Phys. Rev. E 53 (6), 5957-5981.

Grossmann, S. \& Lohse, D. 2000 Scaling in thermal convection: a unifying theory. J. Fluid Mech. 407, 27-56.

Hewitt, J. M., Mckenzie, D. P. \& Weiss, N. O. 1975 a Dissipative heating in convective flows. Journal of Fluid Mechanics 68, 721-738.

Hewitt, J. M., McKenzie, D. P. \& Weiss, N. O. $1975 b$ Dissipative heating in convective flows. J. Fluid Mech. 68 (4), 721-738.

Hurlburt, N. E., Toomre, J. \& Massaguer, J. M. 1984 Two-dimensional compressible convection extending over multiple scale heights. Astrophys. J. 282, 557-573.

Jimenez, J. \& ZufiriA, J. A. 1987 A boundary-layer analysis of Rayleigh-Bénard convection at large Rayleigh number. Journal of Fluid Mechanics 178, 53-71.

LANTZ, S.R. \& FAN, Y. 1999 Anelastic magnetohydrodynamic equations for modeling solar and stellar convection zones. Astrophys. Journal 121, 247-264.

Malkus, W. V. R. 1954 The heat transport and spectrum of thermal turbulence. Proceedings of the Royal Society A 225, 196-212.

Malkus, W. V. R. 1964 Boussinesq equations and convection energetics. report of the Woods Hole Oceanographic Institute 64-66.

McKenzie, DAn \& JARvis, Gary 1980 The conversion of heat into mechanical work by mantle convection. Journal of Geophysical Research: Solid Earth 85 (B11), 6093-6096.

OBerbeck, A. 1879 Über die Wärmeleitung des Flüssigkeiten bei Berücksichtigung des Strömungen infolge von Temperaturdifferenzen. Ann. Phys. Chem. 7, 271-292.

Ogura, Y. \& Phillips, N.A. 1961 Scale analysis of deep and shallow convection in the atmosphere. J. Atm. Sci. 19, 173-179.

Ricard, Y. 2015 Vol. 7, Physics of mantle convection, Treatise on Geophysics. Cambridge University Press.

Spiegel, E. A. \& Veronis, G. 1971 On the boussinesq approximation for a compressible fluid. Astrophys. J. 131, 442-447.

Tilgner, A 2011 Convection in an ideal gas at high Rayleigh numbers. Physical Review E $84(2), 026323$.

Verhoeven, Jan, Wiesehöfer, Thomas \& Stellmach, Stephan 2015 Anelastic versus fully 
compressible turbulent Rayleigh-Bénard convection. The Astrophysical Journal 805 (1), 62.

Verhoogen, John 1980 Energetics of the Earth, pp. 67-89. Washington, D.C.: National Academy Press.

Wu, X. Z. \& Libchaber, A. 1991 Non-Boussinesq effects in free thermal convection. Phys. Rev. A 43, 2833-2839. 